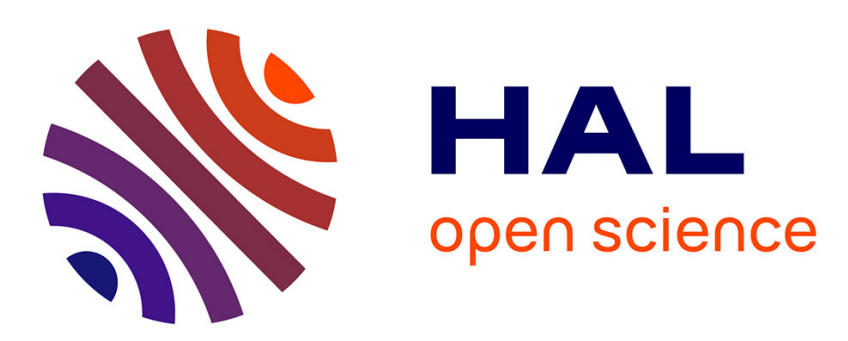

\title{
In-plane mechanics of soft architectured fibre-reinforced silicone rubber membranes
}

\author{
Lucie Bailly, Mamadou Toungara, Laurent Orgéas, Eric Bertrand, Valérie \\ Deplano, Christian Geindreau
}

\section{- To cite this version:}

Lucie Bailly, Mamadou Toungara, Laurent Orgéas, Eric Bertrand, Valérie Deplano, et al.. In-plane mechanics of soft architectured fibre-reinforced silicone rubber membranes. Journal of the mechanical behavior of biomedical materials, 2014, 40, pp.339-353. 10.1016/j.jmbbm.2014.09.012 . hal-01068035

\section{HAL Id: hal-01068035 \\ https://hal.science/hal-01068035}

Submitted on 24 Sep 2014

HAL is a multi-disciplinary open access archive for the deposit and dissemination of scientific research documents, whether they are published or not. The documents may come from teaching and research institutions in France or abroad, or from public or private research centers.
L'archive ouverte pluridisciplinaire HAL, est destinée au dépôt et à la diffusion de documents scientifiques de niveau recherche, publiés ou non, émanant des établissements d'enseignement et de recherche français ou étrangers, des laboratoires publics ou privés. 


\section{Author's Accepted Manuscript}

In-plane mechanics of soft architectured fibre-reinforced silicone rubber membranes

L. Bailly, M. Toungara, L. Orgéas, E. Bertrand, V. Deplano, C. Geindreau

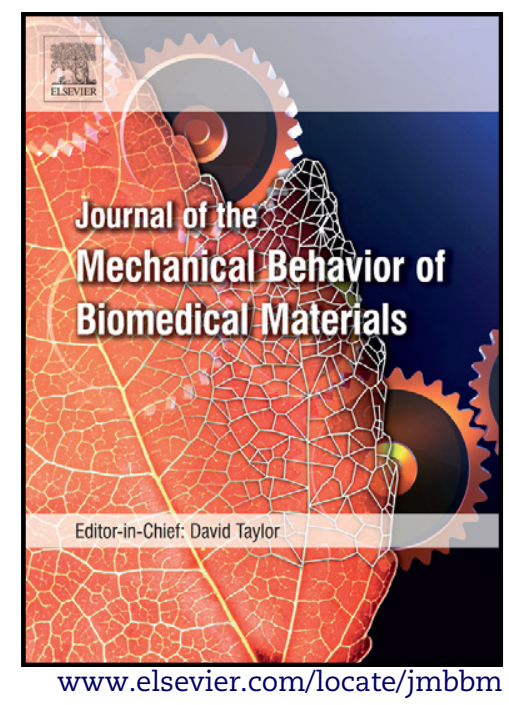

PII: S1751-6161(14)00296-3

DOI: http://dx.doi.org/10.1016/j.jmbbm.2014.09.012

Reference: JMBBM1270

To appear in: Journal of the Mechanical Behavior of Biomedical Materials

Received date:26 June 2014

Revised date: 1 September 2014

Accepted date:

8 September 2014

Cite this article as: L. Bailly, M. Toungara, L. Orgéas, E. Bertrand, V. Deplano, C. Geindreau, In-plane mechanics of soft architectured fibrereinforced silicone rubber membranes, Journal of the Mechanical Behavior of Biomedical Materials, http://dx.doi.org/10.1016/j.jmbbm.2014.09.012

This is a PDF file of an unedited manuscript that has been accepted for publication. As a service to our customers we are providing this early version of the manuscript. The manuscript will undergo copyediting, typesetting, and review of the resulting galley proof before it is published in its final citable form. Please note that during the production process errors may be discovered which could affect the content, and all legal disclaimers that apply to the journal pertain. 


\title{
In-plane mechanics of soft architectured
}

\section{fibre-reinforced silicone rubber membranes}

\author{
L. Bailly ${ }^{\mathrm{a}, *}$, M. Toungara ${ }^{\mathrm{b}, \mathrm{c}}$, L. Orgéas ${ }^{\mathrm{b}, \mathrm{c}}$, E. Bertrand $^{\mathrm{a}}$ \\ V. Deplano ${ }^{a}$ and C. Geindreau ${ }^{b, c}$ \\ a Aix Marseille Université, CNRS, Centrale Marseille, IRPHE UMR 7342, 13384, \\ Marseille, France \\ ${ }^{\mathrm{b}}$ Univ. Grenoble Alpes, 3SR Lab, F-38000 Grenoble, France \\ ${ }^{\mathrm{c}} \mathrm{CNRS}$, 3SR Lab, F-38000 Grenoble, France
}

\begin{abstract}
Silicone rubber membranes reinforced with architectured fibre networks were processed with a dedicated apparatus, allowing a control of the fibre content and orientation. The membranes were subjected to tensile loadings combined with continuous and discrete kinematical field measurements (DIC and particle tracking). These tests show that the mechanical behaviour of the membranes is hyperelastic at the first order. They highlight the influence of the fibre content and orientation on both the membrane in-plane deformation and stress levels. They also prove that for the considered fibrous architectures and mechanical loadings, the motion and deformation of fibres is an affine function of the macroscale transformation. These trends are fairly well described by the micromechanical model proposed recently in Bailly et al. (JMBBM, 2012). This result proves that these materials are very good candidates for new biomimetic membranes, e.g. to improve aortic analogues used for in vitro experiments, or existing textiles used for vascular (endo)prostheses.
\end{abstract}

Key words: Micro-mechanical model, Tensile tests, Biomimetism, Fibre-reinforced membrane, Anisotropy, Material design, Silicone rubber

\footnotetext{
* Corresponding author. Phone: +33 (0)4 135520 27. Address: IRPHE, Technopôle de Château Gombert, 49, rue F. Joliot Curie B.P. 146, 13384 Marseille Cedex 13, France. Email address: lucie.bailly@irphe.univ-mrs.fr (L. Bailly).
} 


\section{Introduction}

Human abdominal aortic tissue (AA) is a complex soft sandwich structure, arranged in three different layers. Within these layers, the distribution of all components displays a double-helix architecture of collagen fibres, elastic fibres, smooth muscle cells and other molecules of the extracellular matrix (Holzapfel, 2008). The fibrous microstructure is characterised by a particular wavy arrangement under stress-free conditions (Freed and Doehring, 2005; Holzapfel, 2008; Rezakhaniha et al., 2011) and by distinctive preferred orientations when subjected to a mechanical loading (Holzapfel, 2006; Horny et al., 2010). These histological features provide the abdominal aortic tissue a highly non-linear, anisotropic and essentially hyperelastic mechanical behaviour (Vande Geest et al., 2006). Over the past decades, several synthetic materials aiming at mimicking the microstructural and/or mechanical specificities of aortic tissue were developed (i) to perform in vitro experiments, or (ii) for bioengineering issues:

(i) In vitro experiments were carried out to characterise biomechanical factors involved in the evolution of arterial diseases such as Abdominal Aortic Aneurysm (AAA). For that purpose, several deformable AAA analogues were processed. Most of them were placed into vascular flow simulators to investigate endovascular repair (Schurink et al., 1998; Flora et al., 2002; Chong and How, 2004; Gawenda et al., 2004). Few were utilised to quantify biological fluid-structure interactions within AAAs (Ene et al., 2011; Deplano et al., 2013, 2014). Inflation tests were conducted by increasing internal pressure in order to identify AAAs deformation or rupture locations (Morris et al., 2004; Doyle et al., 2009, 2010; Corbett et al., 2010; Meyer et al., 2011). However, all these materials were made up of homogeneous elastomers with isotropic material properties, which are very far from the anisotropic hyperelastic behaviour of aortic tissue and from its fibrous microstructure.

(ii) In parallel, in the field of vascular tissue engineering, numerous implants were 
designed so as to be mechanically as close as possible to the host tissue macroscale mechanical behaviour. To this end, polymeric fibrous composites were largely designed as bioinspired scaffolds (Li and Cooper Jr, 2011). Various manufacturing processes were also developed to elaborate fibrous networks (Ratcliffe, 2000; Chen and Hunt, 2007). Currently, a specific interest is provided to fibre electrospinning and textile processing techniques:

(a) Electrospinning technology is favoured to manufacture nano-structured biomaterials made up of filaments close to collagen/elastin fibrils dimensions (Matthews et al., 2002; Boland et al., 2004; Agarwal et al., 2008; Clure et al., 2010). Recent advances allow a better control of the electrospun fibres morphology, strength and size by adjusting set-up conditions or polymer solution parameters (Kowalczyk et al., 2008; Soliman et al., 2011). However, the control of the fibres position and orientation is still a challenging task to generate the desired macroscopic mechanical anisotropy (Bu et al., 2012).

(b) Textile processing techniques are also widely used to develop vascular (endo)prostheses (Jacobs et al., 2003; Chakfe et al., 2004; VanLieshout et al., 2006). Knitted, braided or woven fabrics are characterised by a fine regular arrangement of the fibres, therefore allowing a better control of the macroscopic anisotropic properties (Xu et al., 2010; Schreiber et al., 2010; Yang et al., 2012). However, due to their inappropriate fibrous microstructures, the current commercial textile prostheses do not exhibit suitable deformability (Surovtsova, 2005; Sarkar et al., 2006; Demanget et al., 2012, 2013; Lemercier et al., 2013).

Therefore, the global objective of this work is to design and process new artificial materials, able to mimic the macroscopic anisotropic properties of AA and AAA tissues, their hyperelastic J-shape mechanical response, and their main histological features (fibrous microstructures with distinctive fibre orientations). In the short run, these materials represent appropriate candidates to perform in vitro experiments similar to those carried out in Deplano et al. (2014), with more relevant models of AAs or 
AAAs. In the long run, they could help improving the design of aortic prostheses towards more efficient mechanical performances. To this end, optimal elastomeric fibrous composites were identified in a previous theoretical work using a multi-scale homogenisation process combined with microstructure optimisation (Bailly et al., 2012). The optimised architectured materials are composed of a thin and soft hyperelastic membrane reinforced by periodic fibrous lattices displaying four preferred orientations and hyperelastic fibres. The present study aims at (i) providing experimental advances concerning the manufacturing and mechanical characterisation of such architectured soft fibre-reinforced membranes, and (ii) validating the assumptions stated to build the micro-mechanical model in Bailly et al. (2012). Thus, a summary of the theoretical background is first reported in Section 2. The manufacturing processes of the elastomeric fibrous membranes are presented in Section 3, along with the method used to characterise their mechanical behaviour. Experimental results are detailed in Section 4. A comparison between these results and the prediction of the micromechanical model is proposed and discussed in Section 5.

\section{Theoretical background}

To mimic the main histological features of the arterial tissues, various fibrous architectures were investigated with the micro-mechanical model proposed in Bailly et al. (2012), i.e. single or bi-layers lattices of fibres. As a first step in the validation of the model assumptions, the present study focuses on the one-layer fibrous structure, as illustrated in Figure 1(a). This structure comprises a single lattice of crossed, identical continuous and straight fibres of initial cross-section $S_{0}$, embedded into a thin and soft elastomer matrix. Each fibrous lattice can be seen as a repetition of a Representative Elementary Cell (REC) lying in the $\left(\mathbf{e}_{x}, \mathbf{e}_{y}\right)$ plane, as sketched in Figure 1(b) in the initial configuration $\mathcal{C}_{0}$. The microscale geometrical and mechanical assumptions related to the REC are summarised as follows: 
(i) The REC geometry is characterised by two periodic vectors $\mathbf{P}_{1}=\mathbf{A}_{\mathbf{1}} \mathbf{A}_{\mathbf{4}}=\ell_{0} \mathbf{E}_{1}$ and $\mathbf{P}_{2}=\mathbf{A}_{\mathbf{1}} \mathbf{A}_{\mathbf{2}}=\ell_{0} \mathbf{E}_{2}$ (resp. $\mathbf{p}_{1}=\mathbf{a}_{\mathbf{1}} \mathbf{a}_{\mathbf{4}}=\ell_{1} \mathbf{e}_{1}$ and $\mathbf{p}_{2}=\mathbf{a}_{\mathbf{1}} \mathbf{a}_{\mathbf{2}}=\ell_{2} \mathbf{e}_{2}$ ) joining the extremities $A_{1}, A_{2}$ and $A_{4}$ (resp. $a_{1}, a_{2}$ and $a_{4}$ ) of the REC in the initial $\mathcal{C}_{0}$ (resp. deformed $\mathcal{C}$ ) configuration. The angles $\left(\mathbf{e}_{x}, \mathbf{P}_{1}\right)$ and $\left(\mathbf{e}_{x}, \mathbf{P}_{2}\right)$ [resp. $\left(\mathbf{e}_{x}, \mathbf{p}_{1}\right)$ and $\left.\left(\mathbf{e}_{x}, \mathbf{p}_{2}\right)\right]$ are noted $\theta_{01}$ and $\theta_{02}\left(\right.$ resp. $\theta_{1}$ and $\left.\theta_{2}\right)$ in $\mathcal{C}_{0}(\operatorname{resp}$ in $\mathcal{C})$. Initial REC configuration is defined so that: $\theta_{02}=-\theta_{01}=\theta_{0}$.

(ii) Fibres are supposed to be linked at the REC extremities $A_{1}, A_{2}, A_{3}$ and $A_{4}$, in such a way that the relative displacement of fibres at these points is zero. Between these points, the initial and deformed lengths of fibre $i$ ( $i=1$ and 2 ) are expressed as $\ell_{0 i}^{\mathrm{f}}=\left\|\mathbf{P}_{i}\right\|=\ell_{0}$ and $\ell_{i}^{\mathrm{f}}=\left\|\mathbf{p}_{i}\right\|=\ell_{i}$, respectively. Furthermore, its elongation $\lambda_{i}^{\mathrm{f}}$ and its tension $\mathbf{t}_{i}^{\mathrm{f}}$ are expressed as $\lambda_{i}^{\mathrm{f}}=\ell_{i} / \ell_{0}$ and $\mathbf{t}_{i}^{\mathrm{f}}=t_{i}^{\mathrm{f}} \mathbf{e}_{i}$ (no summation on the index $i$ ). Fibres are assumed to behave as hyperelastic bars, i.e. the tensions $t_{i}^{\mathrm{f}}$ only depend on the fibre elongations $\lambda_{i}^{\mathrm{f}}$ via a fibre strain energy function $W^{\mathrm{f}}$.

(iii) The mechanical interactions between the fibres lattice and the homogeneous elastomer membrane, and between the fibres are negligible.

The macroscale mechanical behaviour of such periodic fibrous lattice was entirely determined analytically, by using the homogenisation of periodic discrete structures (Caillerie et al., 2006; Bailly et al., 2012). It was shown that the overall macroscopic Cauchy tension tensor $\tau^{\mathrm{c}}$ of the one-layer fibrous membrane can be written as:

$$
\boldsymbol{\tau}^{\mathrm{c}}=\boldsymbol{\tau}^{\mathrm{m}}+\frac{1}{\left\|\mathbf{p}_{1} \times \mathbf{p}_{2}\right\|} \sum_{i=1}^{2}\left(\mathbf{t}_{i}^{\mathrm{f}} \otimes \mathbf{p}_{i}\right)
$$

where $\boldsymbol{\tau}^{\mathrm{m}}$ corresponds to the Cauchy tension tensor of the elastomer membrane contribution. Thus, knowing the mechanical behaviour of the elastomeric membrane, that of the fibres, and the geometry of the REC, the mechanical behaviour of the considered architectured and fibre-reinforced membranes is fully estimated: no constitutive parameter has to be fitted a posteriori. 


\section{Experimental procedure}

\subsection{Materials}

Matrix - A biocompatible high strength RTV Silicone elastomer dispersion (PN 40021, Applied Silicone Corporation) was selected as matrix material, due to its high deformability and specific physical properties. It allowed an easy impregnation of the fibrous network and cured at room-temperature. To minimise the scattering of the experimental results, a particular attention was brought to subject the processed membranes to the same curing time $(24 \mathrm{~h})$ and to keep them at the same room temperature (290K) and relative humidity (50\%) until mechanical testing.

The silicone in-plane mechanical response was investigated by means of a uniaxial tensile-testing device (Bose Corporation, Eden Prairie, Minnesota) equipped with a load cell of capacity of $450 \pm 0.01 \mathrm{~N}$. Displacement-controlled tests were performed at room temperature on 8 rectangular samples of mean initial gauge $L_{0}=21.21$ $\mathrm{mm}(\mathrm{STD}=1.07 \mathrm{~mm})$, width $W_{0}=10.13 \mathrm{~mm}(\mathrm{STD}=0.47 \mathrm{~mm})$ and thickness $T_{0}$ $=1.05 \mathrm{~mm}(\mathrm{STD}=0.22 \mathrm{~mm})$, STD being the data standard deviation. Note that these dimensions were determined by photographing the undeformed samples with an accuracy of $0.02 \mathrm{~mm}$. Each sample was subjected to 10 load-unload cycles, with a maximal tensile elongation of 1.30 and an elongation rate of $10^{-2} \mathrm{~s}^{-1}$, representative of AA tissue's physiological loadings (Bailly et al., 2014). During the tests, pictures of the sample's gauge zone were recorded with a JAI CCD camera $(1624 \times 1236$ pixels, $25 \mathrm{~Hz}$ ). Before, speckles were drawn on the surface of each strip so that strain field measurements could be carried out using 2D Digital Image Correlation (DIC), as illustrated in Figure 1(d). Elongations of the specimen along the stretch $\mathbf{e}_{y}$ and the transversal $\mathbf{e}_{x}$ directions, i.e. respectively $\lambda_{y y}$ and $\lambda_{x x}$, were calculated using a DIC software (7D) developed by Vacher et al. (1999). Longitudinal force $F_{y}$ was also measured during the tests and corresponding component of the first Piola-Kirchhoff 
stress tensor was defined as $\Pi_{y y}=F_{y} / W_{0} T_{0}$.

Typical experimental data are displayed in Figure 1(d), showing both the elongation path $\left(\lambda_{x x}, \lambda_{y y}\right)$ and the stress-elongation response $\left(\Pi_{y y}, \lambda_{y y}\right)$. A low hysteresis was measured during the load/unload sequences. Therefore, as a first reasonable approximation, it can be inferred that the considered silicone rubber behaved nearly as a hyperelastic material. Besides, within the investigated elongation range, its mechanical behaviour could be fairly well modelled with a classical neo-Hookean model (Treloar, 1943), thus characterised by a volumetric strain-energy function $W^{\mathrm{m}}=c^{\mathrm{m}}\left(I_{1}-3\right) / 2$. The constant $c^{\mathrm{m}}$ is a positive material parameter, and $I_{1}=\operatorname{tr}(\mathbf{C})$ denotes the first principal invariant of the right Cauchy-Green tensor C. This model was adjusted on both curves, as illustrated in Figure 1(d). For all samples, the average material parameter was found around $c^{\mathrm{m}}=0.24 \mathrm{MPa}(\mathrm{STD}=0.01 \mathrm{MPa})$.

Fibre - A $50 \mu \mathrm{m}$ diameter polyamid monofilament was selected as a fibre candidate. This fibre is commonly used as fishing wire (Feeling 36605, Sensas - 0.8 lb, $0.350 \mathrm{~kg}$ ). Uniaxial tensile tests were performed to characterise the mechanical behaviour of 16 fibre samples cut from this filament on a dedicated testing machine (Instron 3367, $100 \pm 0.01 \mathrm{~N}$ ). Tests were conducted until failure, using an elongation rate of $10^{-2}$ $\mathrm{s}^{-1}$, and an initial fibre length of $200 \mathrm{~mm}$ at rest. The force within each fibre sample $t^{\mathrm{f}}$ was measured during the tests and the corresponding first Piola-Kirchhoff stress was estimated as $\Pi^{\mathrm{f}}=t^{\mathrm{f}} / S_{0}, S_{0}$ being the fibre unloaded cross-section. Figure 1(e) presents the mean stress value $\Pi^{\mathrm{f}}$, as function of fibre elongation $\lambda^{\mathrm{f}}$, measured in average over the 16 samples. The obtained fibre mechanical behaviour is repeatable and non-linear. Failure loads varied from 1.93 to $2.35 \mathrm{~N}$ (mean value $2.21 \mathrm{~N}$ ), and failure stretches ranged between 1.20 and 1.30 (mean value 1.26). Finally, the experimental data could be described by a hyperelastic model proposed in Orgéas et al. (1998) for Shape Memory Alloys (see Figure 1(e)), so that the mean tension within the fibre is expressed as: 


$$
\begin{aligned}
& t^{\mathrm{f}}=\frac{S_{0}}{\lambda^{\mathrm{f}}}\left\{\begin{array}{l}
\frac{\mu_{1}}{2}\left[\epsilon^{\mathrm{f}}+\operatorname{sgn}_{\epsilon}\left\{\epsilon_{s}-\sqrt{\left[\epsilon^{\mathrm{f}}+\operatorname{sgn}_{\epsilon} \epsilon_{s}\right]^{2}+\alpha_{1}^{2}}\right\}\right]+\operatorname{sgn}_{\epsilon} \sigma_{0} \\
+\mu_{2} \epsilon^{\mathrm{f}} \\
+\frac{\mu_{3}}{2}\left[\epsilon^{\mathrm{f}}+\operatorname{sgn}_{\epsilon}\left\{\sqrt{\left[\epsilon^{\mathrm{f}}-\operatorname{sgn}_{\epsilon} \epsilon_{c}\right]^{2}+\alpha_{2}^{2}}-\sqrt{\epsilon_{c}^{2}+\alpha_{2}^{2}}\right\}\right],
\end{array}\right. \\
& \text { with } \quad \epsilon_{s}=\frac{\alpha_{1}^{2}-\left(2 \sigma_{0} / \mu_{1}\right)^{2}}{4 \sigma_{0} / \mu_{1}} \quad \text { and } \quad \operatorname{sgn}_{\epsilon}=\left\{\begin{array}{lll}
1 & \text { if } & \epsilon^{\mathrm{f}} \geq 0 \\
-1 & \text { if } & \epsilon^{\mathrm{f}}<0 .
\end{array}\right.
\end{aligned}
$$

This tension is a function of the logarithmic strain, $\epsilon^{\mathrm{f}}=\ln \lambda^{\mathrm{f}}$, and of 7 constitutive parameters: $\mu_{1}, \mu_{2}, \mu_{3}, \sigma_{0}, \epsilon_{c}, \alpha_{1}$ and $\alpha_{2}$ (Orgéas, 1997). The model parameters were identified from the experimental data given in Figure 1(e) over the stretch range $\lambda^{\mathrm{f}} \in[1: 1.15]$. The latter covers the range of tensile loadings predicted within the fibres in the experiments below. The identification yielded to $\mu_{1}=2444.94$ $\mathrm{MPa}, \mu_{2}=223.16 \mathrm{MPa}, \mu_{3}=38652.62 \mathrm{MPa}, \sigma_{0}=42.18 \mathrm{MPa}, \epsilon_{c}=16.25 \times 10^{-2}$, $\alpha_{1}=9.64 \times 10^{-3}$ and $\alpha_{2}=5.20 \times 10^{-2}$.

\subsection{Architectured soft fibre-reinforced membranes}

Processing route - The as-described fibre and matrix materials were used to manufacture the target fibre-reinforced membranes. For that purpose, a specific device was designed to manufacture lattices of crossed and identical straight fibres, impregnated inside rectangular membranes $(160 \mathrm{~mm} \times 50 \mathrm{~mm})$, as shown in Figure 2(a). The device consists of a pantographic deployable structure and a Teflon ${ }^{\mathrm{TM}}$ rectangular mould. Each arm of the pantograph is equipped with a $1 \mathrm{~mm}$-tooth comb. Three parameters of the device were adjusted before each composite membrane's elaboration:

(i) the number of teeth involved in the manufacturing (see Figure 2(c)). This parameter allows the control of the initial fibre length of the lattice, $\ell_{0}$, and thus, 
the fibre content. Length $\ell_{0}$ can vary by step of $1 \mathrm{~mm}$.

(ii) the angulation of the pantograph, adjustable by translation of the arm's crossjunctions as illustrated in Figure 2(b). This parameter allows the control of the initial half-angle between fibres, $\theta_{0}$. It can be varied between $20^{\circ}$ and $70^{\circ}$ by step of $5^{\circ}$.

(iii) the angle $\beta_{0}$ as defined in Figure 2(c), adjustable by rotation of the mould. This parameter allows to control the orientation of the fibre network in the $\left(\mathbf{e}_{x}, \mathbf{e}_{y}\right)$ plane. Two cases were investigated: $\beta_{0}=\left\{0^{\circ}, 45^{\circ}\right\}$.

At a chosen configuration $\left(\ell_{0}, \theta_{0}, \beta_{0}\right)$, a first unidirectional fibre network was built using the same fibre. The fibre was guided alternatively by two combs of the pantograph facing to each other. In the same way, a second unidirectional network was built using the other combs of the device, and superimposed on the first one. So as to create a $1 \mathrm{~mm}$-thick membrane, the lattice was then embedded by a $20 \mathrm{~m} \ell$-layer of silicone casted into the mould. Note that the depth of the mould was designed (i) to account for the average mass loss (58\%) measured after the silicone curing due to solvent evaporation and shrinkage of the silicone during curing, and (ii) to spatially equilibrate the microstructure along the membrane's thickness. Finally, several composite membranes were processed, characterised by six microstructural arrangements $\left(\ell_{0}, \theta_{0}\right)$ as follows: $\left(1 \mathrm{~mm}, 45^{\circ}\right),\left(3 \mathrm{~mm}, 45^{\circ}\right),\left(1 \mathrm{~mm}, 30^{\circ}\right),(3 \mathrm{~mm}$, $\left.30^{\circ}\right),\left(1 \mathrm{~mm}, 60^{\circ}\right),\left(3 \mathrm{~mm}, 60^{\circ}\right)$. These target values of both parameters were chosen in line with previous predictions found in Bailly et al. (2012). Typical illustrations of manufactured microstructures are presented in Figure 2(d).

Samples - Eight rectangular strips $\left(S_{i}, i \in[1, \ldots, 8]\right)$ were cut from the composite membranes. Their geometrical dimensions were determined so as to preserve the same separation scale from a given sample to another one, by considering 6 RECs along the width and 9 RECs along the length. Strip's initial dimensions were optically measured and summarised in Table 1 . In the following, for the sake of clarity, the composite strip $S_{i}$, characterised by the triplet of parameters $\left(\ell_{0}, \theta_{0}, \beta_{0}\right)$, will be 
denoted as $S_{i}\left(\ell_{0}, \theta_{0}, \beta_{0}\right)$. In practice, the values of $\left(\ell_{0}, \theta_{0}\right)$ slightly differed from the target ones. Thus, optical measurements were processed using a particle-tracking method applied on strips' images registered at rest. The image processing was based on the tracking of experimental REC extremities, as illustrated in Figure 3 upon one REC. For each strip, the measured values of $\left(\ell_{0}, \theta_{0}\right)$ were averaged on all the surface's RECs and noted $\left(\overline{\ell_{0}}, \overline{\theta_{0}}\right)$. Values of $\left(\overline{\ell_{0}}, \overline{\theta_{0}}\right)$ are detailed in Table 1 . The accuracy of the tracking method was assessed at $\left(0.05 \mathrm{~mm}, 0.2^{\circ}\right)$. Finally, the target values of $\left(\ell_{0}, \theta_{0}\right)$ were achieved within a mean precision of $(0.07 \pm 0.05 \mathrm{~mm}, 0.4 \pm$ $0.2^{\circ}$ ) over the whole database.

Tensile tests - The in-plane mechanical response of the $S_{i}$ samples was investigated using the tensile-testing device and the protocol previously described for the homogeneous membrane. The stretch direction was defined by the vector $\mathbf{e}_{y}$. Thus, for $\beta_{0}=0^{\circ}$, the stretch direction matches the REC diagonal $\left(\mathbf{A}_{\mathbf{4}} \mathbf{A}_{\mathbf{2}}\right.$ direction). For $\beta_{0}=45^{\circ}$, the stretch direction matches the fibres one, as illustrated in Figure 3.

Each test was repeated twice in order to ensure its reproducibility, and coupled with image recording of the deformed strip. In the following, focus is given to the first load of each test only. The discrete kinematical field measured at the fibre scale was derived from the tracking of experimental REC nodes $A_{i}$ on each image, as displayed in Figure 3. The image processing was repeated three times on four centered RECs for each composite sample. This yielded to average values of the microscopic longitudinal and transversal elongations, i.e. $\lambda_{y y}$ and $\lambda_{x x}$ respectively. The local value of the half-angle between the fibres was also derived. This angle was noted $\widehat{\theta_{0}}$ and its evolution was calculated along the test. In summary, for each strip $S_{i}$, Table 2 compares (i) the target value of angle $\theta_{0}$, (ii) the angle $\overline{\theta_{0}}$ averaged over all surface's RECs, and (iii) the angle $\widehat{\theta_{0}}$ averaged over the four RECs associate to the measured elongations $\left(\lambda_{x x}, \lambda_{y y}\right)$.

Finally, some samples' surfaces were additionally covered by speckles to perform 2D DIC measurements. In that case, the continuous kinematical field applied on the 
composite membrane was derived in average over a whole REC. Therefore, kinematics measured at the composite scale (global, DIC measurements) and at the fibre scale (local, particle tracking measurements) could be compared.

\section{Experimental results on composite membranes}

\subsection{General trends}

Typical measurements are reported in Figure 3, showing the local loading paths $\left(\lambda_{x x}, \lambda_{y y}\right)$, the derived local half-angle between the fibres $\hat{\theta}$ as a function of the elongation $\lambda_{y y}$, and the corresponding tension-elongation response in the composite membrane $\left(\tau_{y y}, \lambda_{y y}\right)$. Two tests repeated on samples $S_{1}(1,45,0)$ and $S_{2}(1,45,45)$ are illustrated, highlighting important general features:

- whatever the loading angle $\beta_{0}$, the repeatability of each test is ensured.

- the local kinematical fields are optically measurable, with an accuracy on angle $\widehat{\theta}$ being of $0.2^{\circ}$. During the tests, a typical quasi-linear $\hat{\theta}$-variation is observed for $\beta_{0}=0^{\circ}$, against zero $\theta$-variation for $\beta_{0}=45^{\circ}$ (see second row in Figure 3 ). This angle variation comes up to $15^{\circ}$ at the applied maximal strain when $\beta_{0}=$ $0^{\circ}$. Note that for each sample, a slight change in the value of initial angle $\widehat{\theta_{0}}( \pm$ $\left.1 / 2^{\circ}\right)$ is observed between the first test and the second one: this is probably due to possible fibrous rearrangement (e.g. slight rotation of the fibres due to the low hysteresis measured after loading cycles) or local variations in the sample's architecture (see Table 2).

- the mechanical effects of the fibrous reinforcement are highly dependent on the loading direction. For $\beta_{0}=0^{\circ}$, the fibres progressively align with the stretch direction (see pictures in Figure 3(a)). Hence, the lattice mechanical contribution combines the rotations of the fibres with their tensile response. These mechanisms typically yield to a drastic contraction of the sample along the transverse 
direction (up to $\lambda_{x x}=0.7$ ), and low values of the mechanical tension in the overall membrane at $\lambda_{y y}=1.3$ (maximum value of $\tau_{y y}$ below $0.5 \mathrm{~N} / \mathrm{mm}$ ). On the contrary, for $\beta_{0}=45^{\circ}$, the fibres arranged perpendicular to the stretch direction are slightly compressed. They strongly restrain the membrane width's contraction along the transverse direction $\left(\lambda_{x x}>0.97\right.$ during the test). In that case, only the fibres aligned with the loading direction contribute to the overall mechanical tension measured in the composite membrane. Therefore, as the silicone rubber matrix displays soft mechanical properties, the lattice mechanical contribution at the macroscale is nearly equivalent to the composite's one. The lattice contribution also comes to the tensile response of individual fibre, weighted by the volume fraction of the fibres parallel to the loading direction, $v_{f}$. This volume fraction is defined by the ratio of the volume of fibres parallel to the loading direction within one REC in configuration $\mathcal{C}_{0}\left(\pi d_{0}^{2} \ell_{0} / 4\right)$, over the volume of the REC $\left(T_{0} \ell_{0}^{2} \sin \left(2 \theta_{0}\right)\right)$. This ratio yields to $v_{f}=\frac{\pi d_{0}^{2}}{4 T_{0} \ell_{0} \sin \left(2 \theta_{0}\right)}$, i.e. $0.2 \%$ for $S_{2}(1,45,45)$.

\subsection{Comparison between global and local kinematics}

Figure 4(a) confronts the global kinematics measured at the composite scale by 2D DIC, with the local kinematics measured at the fibre scale by tracking the displacements of the REC nodes $A_{i}$. Samples $S_{3}(3,45,0)$ and $S_{4}(3,45,45)$ were chosen for illustration. Whatever the loading direction, a fairly good quantitative agreement was measured between global and local kinematics, as shown by the superimposition of the loading paths derived from both techniques in Figure 4(a). In particular, the relative difference $e_{r}$ between a couple of stretches determined by DIC $\left(\lambda_{x x}^{D I C}, \lambda_{y y}^{D I C}\right)$, and the one determined by particle tracking $\left(\lambda_{x x}^{t}, \lambda_{y y}^{t}\right)$ was calculated using:

$$
e_{r}=\frac{\left[\left(\lambda_{x x}^{D I C}-\lambda_{x x}^{t}\right)^{2}+\left(\lambda_{y y}^{D I C}-\lambda_{y y}^{t}\right)^{2}\right]^{0.5}}{\left[\left(\lambda_{x x}^{t}-1\right)^{2}+\left(\lambda_{y y}^{t}-1\right)^{2}\right]^{0.5}}
$$


Regarding data series with $\beta_{0}=45^{\circ}$, the average value, $\overline{e_{r}}$, comes to $13.8 \%$ for $\lambda_{y y} \in[1: 1.14]$, and drops down to $6 \%$ for $\lambda_{y y}>1.05$. Regarding data series with $\beta_{0}=0^{\circ}, \overline{e_{r}}$ comes to $17.7 \%$ for $\lambda_{y y} \in[1: 1.23]$. It falls below $15 \%$ for $\lambda_{y y}>1.05$. Note that this quantitative agreement is nearly unchanged when calculating the global kinematical field in average over a surface of $3 \times 4$ RECs (not shown). From these results, the overall membrane and the REC were considered to be subjected to the same macroscopic transformation gradient (see the discussion below). In the following, the discussed loading paths $\left(\lambda_{x x}, \lambda_{y y}\right)$ refer exclusively to the elongations calculated at the fibre scale.

\subsection{Influence of the loading angle $\beta_{0}$}

The graphs of Figure 5(a) compare the loading paths and the Cauchy tensionelongation responses measured on three samples: the isotropic homogeneous silicone membrane, samples $S_{1}(1,45,0)$ and $S_{2}(1,45,45)$. All samples are characterised by a similar initial thickness (relative STD being 6.7\%), which enables a comparison relying on tension curves.

Both the mechanical structural reinforcement and the anisotropy induced by the fibrous lattice are emphasised in the left graph of Figure 5(a). For the silicone membrane, the shrinkage of the sample width is about $10 \%$ at $\lambda_{y y}=1.25$. For composite $S_{1}(1,45,0)$, the progressive alignment of the fibres along the loading direction favours the sample contraction along the $x$-direction, and yields to a width-shrinkage of about $30 \%$ at $\lambda_{y y}=1.25$. On the contrary, for sample $S_{2}(1,45,45)$, the width's decrease is restrained to about $2 \%$ beyond $\lambda_{y y}=1.05$.

The anisotropy is also highlighted in the right graph of Figure 5(a), showing a global stiffening of the mechanical behaviour induced by the fibrous lattice. The more the fibres are aligned along the stretch direction, the higher the stiffness of the sample and stress levels. A tangent modulus was assessed at a tension value typically 
measured in aortic tissue for tensile loadings (Vande Geest et al., 2006; Bailly et al., 2012), i.e. $\tau_{y y}=0.1 \mathrm{~N} / \mathrm{mm}$. The stiffening was characterised by a modulus of 1.13 $\mathrm{N} / \mathrm{mm}$ for $S_{1}(1,45,0)$ [resp. $19.76 \mathrm{~N} / \mathrm{mm}$ for $\left.S_{2}(1,45,45)\right]$, thus increased by a ratio 1.3 (resp. 22.5) in comparison to the silicone case $(0.88 \mathrm{~N} / \mathrm{mm}$ ). At last, the mechanical responses of the silicone and the sample $S_{1}(1,45,0)$ were initially superimposed. This suggests that, for this fibrous architecture, i.e. for $\beta_{0}=0^{\circ}$ and for values of $\theta_{0}$ below $45^{\circ}$, the contribution of the lattice is negligible, at least for $\lambda_{y y}<1.05$

\subsection{Influence of the initial fibre length $\ell_{0}$}

The graphs of Figure 5(b) compare the loading paths and the tension-elongation responses measured on five samples of comparable initial thickness (relative STD being $13.7 \%$ ): the silicone membrane, samples $S_{1}(1,45,0), S_{2}(1,45,45), S_{3}(3$, $45,0)$ and $S_{4}(3,45,45)$. The influence of initial fibre length $\ell_{0}$ can be assessed by comparing the mechanical response of $S_{1}(1,45,0)$ and $S_{3}(3,45,0)$, and that of $S_{2}(1,45,45)$ and $S_{4}(3,45,45)$.

Regarding the elongation paths shown in Figure 5(b), the influence of the initial fibre length is noticeable when the loading direction is not aligned with the fibres ones. Indeed, for $\beta_{0}=0^{\circ}$, the contraction of the sample initial width $\left(15 \%\right.$ for $\left.\lambda_{y y}=1.25\right)$ is twice lower for $\ell_{0}=3 \mathrm{~mm}$ than that observed for $\ell_{0}=1 \mathrm{~mm}$. By contrast, for $\beta_{0}=45^{\circ}$, the impact of fibre length $\ell_{0}$ on the sample contraction is negligible.

Regarding the tension-elongation responses shown in Figure 5(b), all composites exhibit a global stiffening as compared to the silicone membrane, except for case $S_{3}(3,45,0)$. The similarity between the responses of $S_{3}(3,45,0)$ and the silicone is mainly ascribed to the differences in thickness samples (ratio of values being 0.76). This similarity disappears when tension data are converted into first PiolaKirchhoff stresses (not shown). For a given stretch direction $\beta_{0}$, the samples with 
higher volume fraction of fibres (i.e. for $\ell_{0}=1 \mathrm{~mm}$ ) are stiffer. In particular, the ratio of the tensions achieved for $S_{4}(3,45,45)$ by the ones obtained for $S_{2}(1,45,45)$ varies between 0.20 and 0.41 , around a mean value of 0.36 . Beyond low elongations $\left(\lambda_{y y}>1.03\right)$, this ratio rises up to 0.39 in average. This value comes close to the ratio of the volume fractions of the fibres parallel to the loading direction within each sample (i.e. 0.40).

\subsection{Influence of the initial half-angle between fibres $\theta_{0}$}

The graphs plotted in Figure 5(c) compare the loading paths and the tensionelongation responses measured on five samples of similar initial thickness (relative STD being $8.2 \%$ ) for various values of $\theta_{0}$. All composite samples are characterised by an identical initial fibre length $\left(\ell_{0}=1 \mathrm{~mm}\right)$ and the same loading direction $\left(\beta_{0}=0^{\circ}\right)$.

For $\theta_{0}=30^{\circ}$, the decrease of the sample's width is very close to the one of the matrix alone, as shown in the left graph of Figure 5(c). Tension-elongation responses are also rather similar. These results imply that, for such lattice architectures and such elongation range, the fibres are not loaded and they weakly disturb the deformation of the silicone.

By contrast, for $\theta_{0}=60^{\circ}$, the fibres tend to align along the loading direction as soon as $\lambda_{y y}>1$. Therefore, the reduction of the sample width is much higher than in the

previous case $\left(\theta_{0}=30^{\circ}\right): \lambda_{x x}=0.68$ versus $\lambda_{x x}=0.90$ for $\lambda_{y y}=1.25$. Furthermore, the elongation paths being similar for cases $\theta_{0}=45^{\circ}$ and $\theta_{0}=60^{\circ}$, the influence of the fibres' initial orientation is clearly demonstrated on the corresponding tensionelongation responses. The more the fibres are aligned along the stretch direction, the higher the stiffness of the sample.

Note that above results are consistent with those obtained by comparing samples 
$S_{3}(3,45,0), S_{7}(3,60,0)$ and $S_{8}(3,30,0)$ exhibiting a larger initial fibre length, $\ell_{0}=$ $3 \mathrm{~mm}$ (see Figure 6). In particular, the low tension-elongation response measured for $S_{8}(3,30,0)$ is linked with the small thickness of the sample as compared to the matrix (ratio of values being 0.67 , i.e. the lowest in the database).

\section{Validation of the micro-mechanical model}

\subsection{Relevance of some of the model assumptions}

As detailed in sections 2 and 3, some major microscale geometrical and mechanical assumptions were formulated in order to apply the micro-mechanical model. Several experimental observations gained from results shown in section 4 ensure the relevance of these assumptions:

(i) For the tensile loadings under study, the REC geometry is assumed to be diamond-shaped in the initial (resp. actual) configuration $\mathcal{C}_{0}$ (resp. $\mathcal{C}$ ). Besides, the displacements of nodes $A_{i}$ are assumed to be affine functions of the macroscale gradient of the geometrical transformation the samples are subjected to.

The tracking of the experimental RECs extremities showed that their diamond pattern remained topologically unaffected by the transformation (see Figure 3). Besides, Figure 4 also proved that the motion of nodes $A_{i}$ were identical to the displacement field recorded at the macroscale with the DIC technique. These observations thus validate above assumptions.

(ii) The fibre mechanical behaviour is predicted by a hyperelastic model (Eq. 2), adjusted on experimental tensile tests performed on fibres (Figure 1(d)). This model assumes a symmetrical behaviour under compressive loading.

It was shown that the fibres are subjected to compressive loading for cases 
with $\beta_{0}=45^{\circ}$. No buckling effect was observed during the considered tests (because the compressive strains remained small and because compressed fibres are confined within the matrix). Thus, the above assumption is reasonable for the considered configurations.

(iii) Finally, the mechanical interactions between the fibres and the matrix, and between the fibres forming the lattice are assumed negligible. Therefore, at the first order, the tension decomposition in Eq. (1) can be considered as a reasonable approximation of the membrane mechanics.

The relevance of this last assumption is detailed in the following.

\subsection{Comparison between experimental data and theoretical predictions}

Theoretical predictions derived from Eq. (1) were confronted to the experimental results presented in section 4, and displayed along in Figures 5 and 6. All these predictions were calculated by prescribing the experimental elongation paths $\left(\lambda_{x x}, \lambda_{y y}\right)$ as input loadings, instead of using an additional assumption of simple tensile test conditions. Note that each plotted experimental elongation path was associated to a local value of the initial half-angle between fibres, $\widehat{\theta_{0}}$ (see bold values in Table 2 ). Therefore, in the following, the comparison between experiments and predictions is focused on the Cauchy tension - elongation curves $\left(\tau_{y y}, \lambda_{y y}\right)$.

Over the whole database, whatever the considered triplet of parameters $\left(\ell_{0}, \theta_{0}, \beta_{0}\right)$, Figures 5 and 6 demonstrate that the micro-mechanical model predictions fairly well fit the experimental data (cases of discrepancies observed in the database are detailed thereafter). This result is obtained despite the model simplicity and bearing in mind that none arbitrary macroscale adjustment was made a posteriori, i.e. all the model data were acquired at the microscale (the REC scale). In particular, the model is able to predict very precisely the mechanical behaviour of composites $S_{1}(1$, $45,0), S_{3}(1,45,0)$ and $S_{6}(1,30,0)$. The average relative difference between data 
and predicted tensions is equal to $5.8 \%, 9.5 \%$ and $9.7 \%$ respectively. This mean difference remains below $20 \%$ for most other composites, with a value of $11.7 \%$ for $S_{2}(1,45,45), 16.4 \%$ for $S_{4}(3,45,45), 18.7 \%$ for $S_{7}(3,60,0)$ and $15.8 \%$ for $S_{8}(3$, $30,0)$. Note that this quantitative agreement between predictions and experimental data is similar when considering the second local value of $\widehat{\theta_{0}}$ in Table 2 (not shown). Such a good accordance validates model assumption (iii).

However, some discrepancies can be observed in two particular cases:

- the first case is pointed out in Figure 7(a). Two tests were performed on sample $S_{1}(1,45,0)$, with a good repeatability in both loading path and tensionelongation curves (see Figure 3(a)). The unique input difference between both test conditions relied on the applied experimental path and the associate value of initial angle $\widehat{\theta_{0}}$, as measured to determine the local kinematical field. The local angle $\widehat{\theta_{0}}$ was assessed to $47.5^{\circ}$ in one case, and to $46.4^{\circ}$ in the other (see Table 2). The impact of the weak difference between both $\widehat{\theta_{0}}$-values on the macroscopic tension $\tau_{y y}$ is illustrated in Figure 7(a), along with the model predictions (solid lines). The load $t^{\mathrm{f}}$ predicted within REC fibre $i(i=1$ and 2$)$ in each case is plotted in Figure $7(b)$. The elongations $\lambda^{\mathrm{f}}$ predicted at the fibre scale are also plotted as function of the macroscopic loading $\lambda_{y y}$ in Figure 7 (c). Thus, it is shown that a very small change in the lattice fibrous architecture can induce very different tensile loadings at the fibre scale: in case $\widehat{\theta_{0}}=46.4^{\circ}$, the fibre elongation $\lambda^{\mathrm{f}}$ stayed close to 1 up to $\lambda_{y y} \in[1: 1.05]$. This implies that the fibres in the REC were subjected to tension later than the ones of the sample with $\widehat{\theta_{0}}=47.5^{\circ}$. Such a delayed stretching altered the overall mechanical behaviour of the composite in a noticeable manner. From this point of view, the initial half-angle between fibres appears as a critical parameter in case of loading direction far from the fibre orientation.

- the second important discrepancy of the database is highlighted in Figure 7(d). 
Two tests were performed on sample $S_{5}(1,60,0)$, with a good repeatability in both loading path (not shown) and tension-elongation curves. Again, the unique difference between both tests relied on the measurement of the local angle $\widehat{\theta_{0}}$, i.e. $61.9^{\circ}$ in one case, and $62.7^{\circ}$ in the other. The impact predicted on the macroscopic tension $\tau_{y y}$, the fibre load $t^{\mathrm{f}}$ and the fibre elongation $\lambda^{\mathrm{f}}$ is displayed in Figures $7(\mathrm{~d}), 7(\mathrm{e})$ and $7(\mathrm{f})$ respectively. In that lattice configuration, both theoretical predictions are rather close to each other despite the $1-2^{\circ}$ of difference in $\widehat{\theta_{0}}$ assessment. The REC fibres were subjected to tension from the beginning of the test $\left(\lambda^{\mathrm{f}}>1\right.$ once $\lambda_{y y}>1$ in each case). Thus, for comparable macroscopic loadings (e.g. $\left.\lambda_{y y}=1.18\right)$, the load achieved within the REC fibres is predicted four times greater than in previous lattice configuration $\left(\widehat{\theta_{0}} \approx 45^{\circ}\right)$, as shown when comparing Figures $7(\mathrm{e})$ and $7(\mathrm{~b})$. However, the predictions largely deviate from the experimental tensions (mean relative difference being $86.7 \%$ ). These discrepancies could be induced either by local stress heterogeneities generated within the composite, or by a misalignment of the sample's microstructure with respect to the load direction.

\section{Conclusion}

The process of artificial membranes able to mimic the mechanical and histological specificities of aortic tissue is needed to perform in vitro experiments as carried out in Deplano et al. (2014), using more relevant models of aortic wall. This will help improving the current knowledge on AAA evolution, and can also contribute to bioengineering challenges (e.g. building aligned and orderly fibrous structures with a controlled positioning, improving the deformability of future prostheses). In this global context, the present study proposed an original technical device to design architectured soft-fibre reinforced membranes. With non-linear, anisotropic mechanical properties at the macroscale, and favoured fibrous orientations at the microscale, these membranes represent appropriate candidates for biomimetic goals. 
The control of a macroscale mechanical anisotropy was allowed only by changing two microstructural parameters: the initial half-angle between the fibres $\theta_{0}$, and the fibre length $\ell_{0}$. The particular case of a single lattice of crossed, identical and straight monofilaments of $50 \mu \mathrm{m}$ in diameter, embedded into a $1 \mathrm{~mm}$-thick soft matrix in silicone was investigated in this work. Eight fibrous membranes of different microstructures were manufactured. The target values of $\left(\ell_{0}, \theta_{0}\right)$ were achieved within a mean precision of $\left(0.07 \pm 0.05 \mathrm{~mm}, 0.4 \pm 0.2^{\circ}\right)$ over the whole database. The fibrous membranes were then characterised using a tensile testing device, for two loading directions $\beta_{0}$. Optical recordings of displacement fields were realised at both macro-and microscale. These experiments allowed the validation of the assumptions of the micro-mechanical model presented in a previous work (Bailly et al., 2012), and based on the homogenisation of periodic discrete structures. This study demonstrates that the model is able to describe the experimental database in a good quantitative agreement, whatever the triplet of parameters $\left(\ell_{0}, \theta_{0}, \beta_{0}\right)$.

The present experimental work must be completed by new biaxial tensile loadings, in order to assess the micro-mechanical model for loadings closer to the ones that arterial tissues are subjected to. Further tests will be coupled with 3D DIC measurements, in order to capture possible out-of-plane displacement fields of the deformed membranes. Finally, manufacturing of wavy substructures in the fibrous lattice would allow the biomimetism of the membranes, as expected in the target solution identified in Bailly et al. (2012).

Acknowledgements - The authors would like to thank A. Lemercier for her contribution to the experimental work. They gratefully acknowledge the CNRS, the Univ. Joseph Fourier and the Institute Carnot PolyNat for their financial support. The laboratory 3SR is part of the LabEx Tec 21 (Investissements d'Avenir - grant agreement ANR-11-LABX- 0030). IRPHE institute is part of the LabEx MEC. 


\section{References}

Agarwal, S., Wendorff, J. H. and Greiner, A. (2008), 'Use of electrospinning technique for biomedical applications', Polymer 49, 5603?5621.

Bailly, L., Deplano, V., Lemercier, A., Boiron, O. and Meyer, C. (2014), 'New experimental protocols for tensile testing of abdominal aortic analogues', Medical Engineering 6 Physics. DOI:10.1016/j.medengphy.2014.02.005.

Bailly, L., Geindreau, C., Orgéas, L. and Deplano, V. (2012), 'Towards a biomimetism of abdominal healthy and aneurismal arterial tissues', J Mech Behavior Biomed Mater 10, 151-65.

Boland, E., Matthews, J. A., Pawlowski, K. J., Simpson, D. G., Wnek, G. E. and Bowlin, G. L. (2004), 'Electrospinning collagen and elastin : preliminary vascular tissue engineering', Frontiers in Bioscience 9, 1422-32.

Bu, N., Huang, Y., Wang, X. and Yin, Z. (2012), 'Continuously tunable and oriented nanofiber direct-written by mechano-electrospinning', Materials and Manufacturing Processes 27, 1318-1323.

Caillerie, D., Mourad, A. and Raoult, A. (2006), 'Discrete homogenization in graphene sheet modeling', Journal of Elasticity 84(1), 33-68.

Chakfe, N., Dieval, F., Riepe, G., Mathieu, D., Zbali, I., Thaveau, F., Heintz, C., Kretz, J. and Durand, B. (2004), 'Influence of the textile structure on the degradation of explanted aortic endoprostheses', Euro J Vascular Endovascular Surg. $27,33-41$.

Chen, R. and Hunt, J. A. (2007), 'Biomimetic materials processing for tissueengineering processes', J Mater Chem 17, 3974-3979.

Chong, C. K. and How, T. V. (2004), 'Flow patterns in an endovascular evg for abdominal aortic aneurysm repair', J Biomech 37, 89-97.

Clure, M. M., Sell, S., Simpson, D., Walpoth, B. H. and Bowlin, G. L. (2010), 'A three-layered electrospun matrix to mimic native arterial architecture using polycaprolactone, elastin, and collagen: A preliminary study', Acta Biomater 6, 242233. 
Corbett, T. J., Doyle, B. J., Callanan, A., Walsh, M. T. and McGloughlin, T. M. (2010), 'Engineering silicone rubbers for in vitro studies: Creating aaa models and ilt analogues with physiological properties', Journal of Biomechanical Engineering 132, 0110081-9.

Demanget, N., Avril, S., Badel, P., Orgéas, L., Geindreau, C., Albertini, J. and Favre, J. (2012), 'Computational comparison of the bending behaviour of aortic stent-grafts', J Mech Behavior Biomed Mater 5, 272-82.

Demanget, N., Duprey, A., Badel, P., Orgéas, L., Avril, S., Geindreau, C., Albertini, J. and Favre, J. (2013), 'Finite element analysis of the mechanical performances of 8 marketed aortic stent-grafts', J Endovasc Ther 20, 523-535.

Deplano, V., Knapp, Y., Bailly, L. and Bertrand, E. (2014), 'Flow of a blood analogue fluid in a compliant abdominal aortic aneurysm model: experimental modelling', Journal of Biomechanics 47, 1262-9.

Deplano, V., Meyer, C., Guivier-Curien, C. and Bertrand, E. (2013), 'New insights into the understanding of flow dynamics in an in vitro model for abdominal aortic aneurysms', Medical Engineering and Physics 35, 800-809.

Doyle, B. J., Cloonan, A. J., Walsh, M. T., Vorp, D. A. and McGloughlin, T. M. (2010), 'Identification of rupture locations in patient-specific abdominal aortic aneurysms using experimental and computational techniques', Journal of Biomechanics 43(7), 1408-1416.

Doyle, B. J., Corbett, T. J., Cloonan, A. J., O'Donnell, M. R., Walsh, M. T., Vorp, D. A. and McGloughlin, T. M. (2009), 'Experimental modelling of aortic aneurysms: Novel applications of silicone rubbers', Medical Engineering \& 3 Physics 31, 1002-1012.

Ene, F., Gachon, C., Delassus, P., Carroll, R., Stefanov, F., O'Flynn, P. and Morris, L. (2011), 'In vitro evaluation of the effects of intraluminal thrombus on abdominal aortic aneurysm wall dynamics', Med. Eng. Physics 33(8), 957-966.

Flora, H. S., Talei-Faz, B., Ansdell, L., Chaloner, E. J., Sweeny, A., Grass, A. and Adiseshiah, M. (2002), 'Aneurysm wall stress and tendency to rupture are fea- 
tures of physical wall properties: An experimental study', Journal of Endovascular Therapy 9(5), 665-675.

Freed, A. D. and Doehring, T. C. (2005), 'Elastic response of crimped collagen fibrils', J Biomech Eng 127(4), 587-93.

Gawenda, M., Knez, P., Winter, S., Jaschke, G., Wassmer, G., Schmitz-Rixen, T. and Brunkwall, J. (2004), 'Endotension is influenced by wall compliance in a latex aneurysm model', Eur J Vasc Endovasc Surg 27, 45-50.

Holzapfel, G. (2006), 'Determination of material models for arterial walls from uniaxial extension tests and histological structure', Journal of Theoretical Biology 238, 290-302.

Holzapfel, G. A. (2008), Collagen in arterial walls: Biomechanical aspects, SpringerVerlag, chapter 11, pp. 285-324.

Horny, L., Kronek, J., Chlup, H., Zitny, R., Vesely, J. and Hulan, M. (2010), 'Orientations of collagen fibers in aortic histological section', Bulletin of applied mechanics 6(22), 25-29.

Jacobs, T., Won, J., Gravereaux, E. C., Faries, P. L., Morrissey, N., Teodorescu, V. J., Hollier, L. H. and Marin, M. (2003), 'Mechanical failure of prosthetic human implants: A 10-year experience with aortic stent graft devices', J Vascular Surg $37,16-26$.

Kowalczyk, T., Nowicka, A., Elbaum, D. and Kowalewski, T. (2008), 'Electrospinning of bovine serum albumin. optimization and the use for production of biosensors', Biomacromolecules 9, 2087-2090.

Lemercier, A., Bailly, L., Geindreau, C., Toungara, M., Latil, P., Orgéas, L., Deplano, V. and Boucard, N. (2013), 'Comparison between the mechanical behaviour of the human healthy aa and commercial prostheses under various mechanical loadings', Computer Methods in Biomechanics and Biomedical Engineering 16, 315-1.

Li, W.-J. and Cooper Jr, J. A. (2011), Biomaterials for Tissue Engineering Applications: A Review of the Past and Future Trends, Springer-Verlag, chapter Fibrous Scaffolds for Tissue Engineering, pp. 47-73. 
Matthews, J., Wnek, G., Simpson, D. and Bowlin, G. (2002), 'Electrospinning of collagen nanofibers', Biomacromolecules 3, 232-238.

Meyer, C., Bertrand, E., Boiron, O. and Deplano, V. (2011), 'Stereoscopically observed deformations of a compliant abdominal aortic aneurysm model', Journal of Biomechanics Engineering 133, 11004.

Morris, L., O’Donnell, P., Delassus, P. and McGoughlin, T. (2004), 'Experimental assessment of stress patterns in abdominal aortic aneurysms using the photoelastic method', Strain 40, 165-172.

Orgéas, L. (1997), Etude expérimentale et numérique du comportement thermomécanique d'un alliage à mémoire de forme industriel NiTi, PhD thesis, Université Joseph Fourier - Grenoble 1.

Orgéas, L., Favier, D. and Rio, G. (1998), 'Déformation superélastique non homogène d'une éprouvette de traction de NiTi: expérience et modélisation numérique', $R e$ vue Européenne des Eléments Finis 7, 111-36.

Ratcliffe, A. (2000), 'Tissue engineering of vascular grafts', Matrix Biology 19, 353357.

Rezakhaniha, R., Agianniotis, A., Schrauwen, J. T. C., Griffa, A., Sage, D., Bouten, C. V. C., van de Vosse, F. N., Unser, M. and Stergiopulos, N. (2011), 'Experimental investigation of collagen waviness and orientation in the arterial adventitia using confocal laser scanning microscopy', Biomech Model Mechanobiol. DOI 10.1007/s10237-011-0325-Z.

Sarkar, S., Salacinski, H., Hamilton, G. and Seifalian, A. (2006), 'The mechanical properties of infrainguinal vascular bypass grafts: Their role in influencing patency', Eur J Vasc Endovasc Surg 31, 627-636.

Schreiber, F., Schuster, P., Borinski, M., Vogt, F., Blindt, R. and Gries, T. (2010), 'Improving the mechanical properties of braided shape memory polymer stents by heat setting', AUTEX Research Journal 10, 73-76.

Schurink, G., M. Aarts, N., Wilde, J., van Baalen, J., Chuter, T., Kool, L. and van Bockel, J. (1998), 'Endoleakage after stent-graft treatment of abdominal 
aneurysm: Implications on pressure and imaging - an in vitro study', Journal of Vascular Surgery 28(2), 234-41.

Soliman, S., Sant, S., Nichol, J., Khabiry, M., Traversa, E. and Khademhosseini, A. (2011), 'Controlling the porosity of fibrous scaffolds by modulating the fiber diameter and packing density', Journal of Biomedical Materials Research Part A 96A, 566-574.

Surovtsova, I. (2005), 'Effects of compliance mismatch on blood flow in an artery with endovascular prosthesis', J Biomech 38, 2078-86.

Treloar, L. R. G. (1943), 'The elasticity of a network of long chain molecules (i and ii)', Trans. Faraday Soc. 39, 36-64; 241-246.

Vacher, P., Dumoulin, S., Morestin, F. and Mguil-Touchal, S. (1999), Bidimensional strain measurement using digital images, in 'Proc. Mech. Engrg.', Vol. 213, pp. 811-817.

Vande Geest, J. P., Sacks, M. S. and Vorp, D. A. (2006), 'The effects of aneurysm on the biaxial mechanical behavior of human abdominal aorta', Journal of Biomechanics 39, 1324-1334.

VanLieshout, M., Vaz, C., Rutten, M., Peters, G. and Baaijens, F. (2006), 'Electrospinning versus knitting: two scaffolds for tissue engineering of the aortic valve', J. Biomater. Sci. Polymer 17, 77-8.

Xu, W., Zhou, F., Ouyang, C., Ye, W., Yao, M. and Xu, B. (2010), 'Mechanical properties of small-diameter polyurethane vascular grafts reinforced by weft-knitted tubular fabric', J Biomed Mater Res 92A, 1-8.

Yang, H., Zhu, G., Zhang, Z., Wang, Z., Fang, J. and Xu, W. (2012), 'Influence of weft-knitted tubular fabric on radial mechanical property of coaxial three-layer small-diameter vascular graft', J Biomed Mater Res 100B, 342-349. 


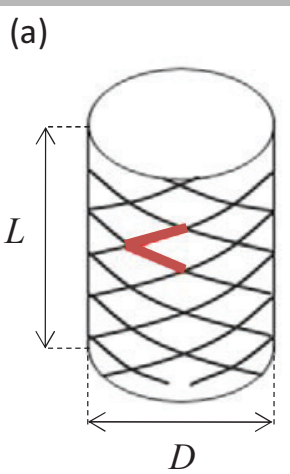

(b)

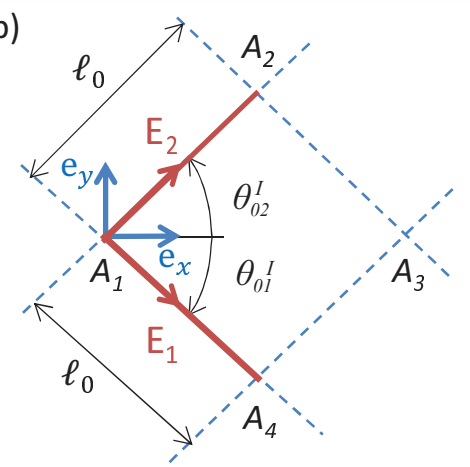

(c)

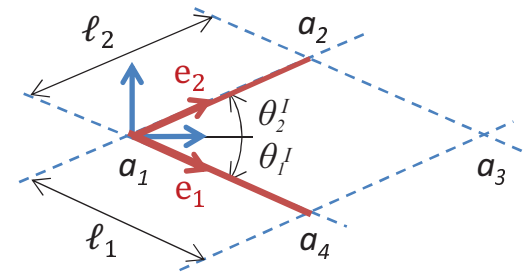

(d)
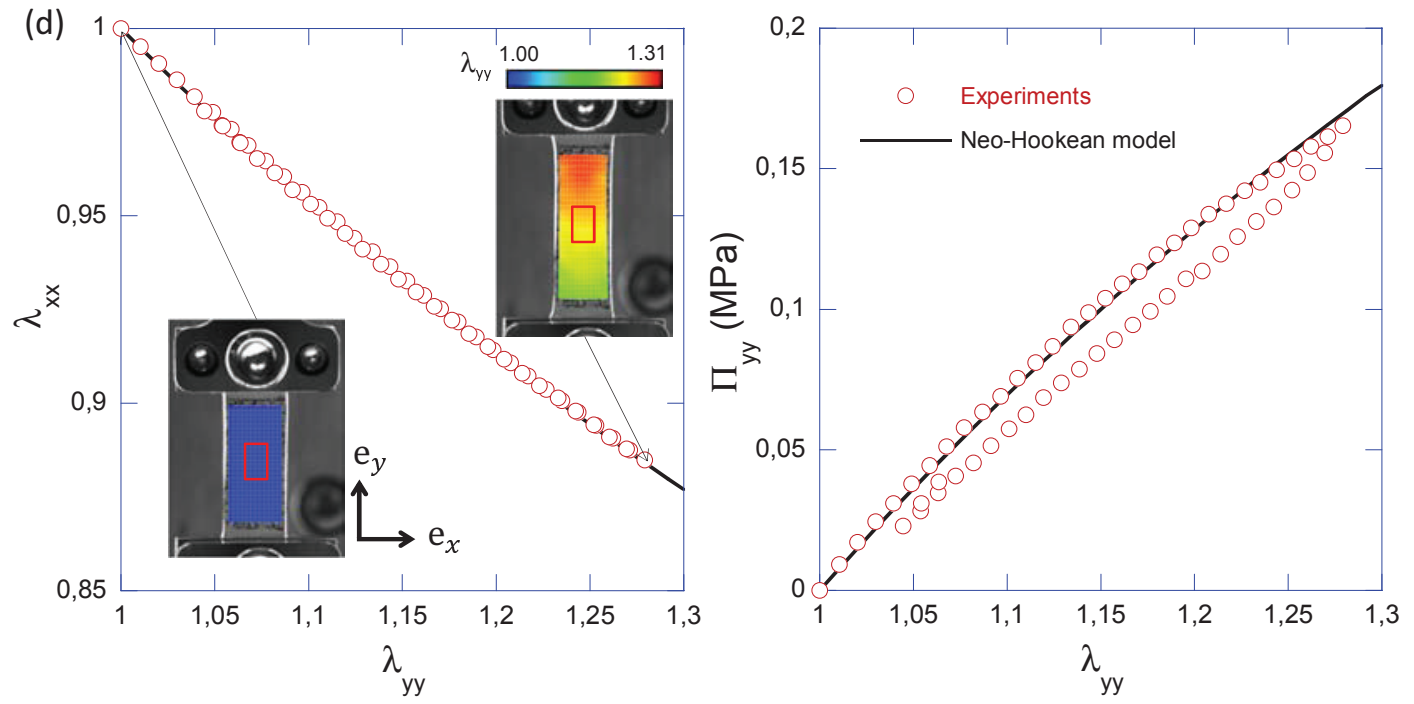

(e)

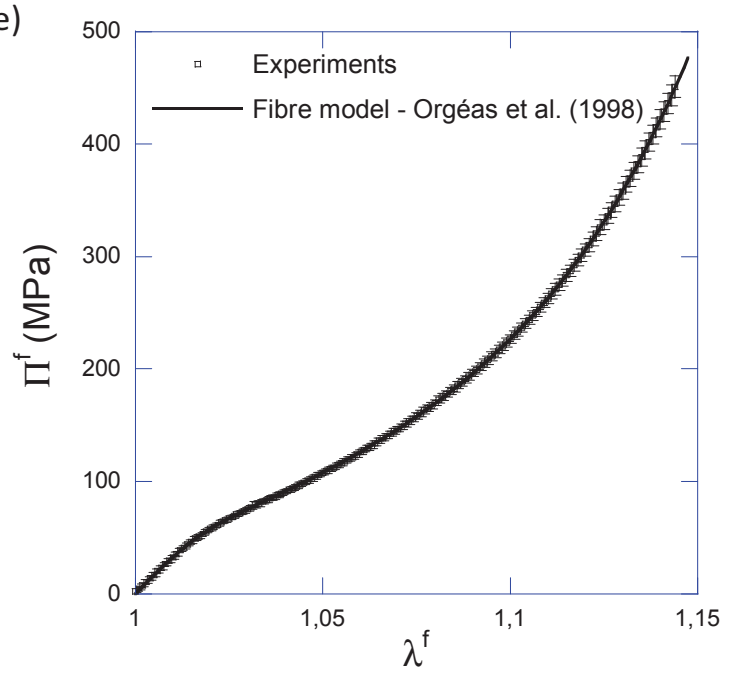

Figure 1. (a) Rhombic lattice and corresponding periodic Representative Elementary Cell of the one-layer fibrous structure: (b) initial undeformed configuration $\mathcal{C}_{0}$, (c) actual configuration $\mathcal{C}$. (d) Mechanical behaviour of the silicone matrix: typical elongation path $\left(\lambda_{x x}, \lambda_{y y}\right)$ and corresponding nominal stress $\Pi_{y y}$ versus elongation $\lambda_{y y}$. The continuous line gives the prediction of the neo-Hookean ${ }_{6}$ model with $c^{\mathrm{m}}=0.24 \mathrm{MPa}$. (e) Mechanical behaviour of the fibre: mean nominal stress $\Pi^{\mathrm{f}}$ versus fibre elongation $\lambda^{\mathrm{f}}$ (average value over 16 samples). Error bars represent the data standard deviation. 


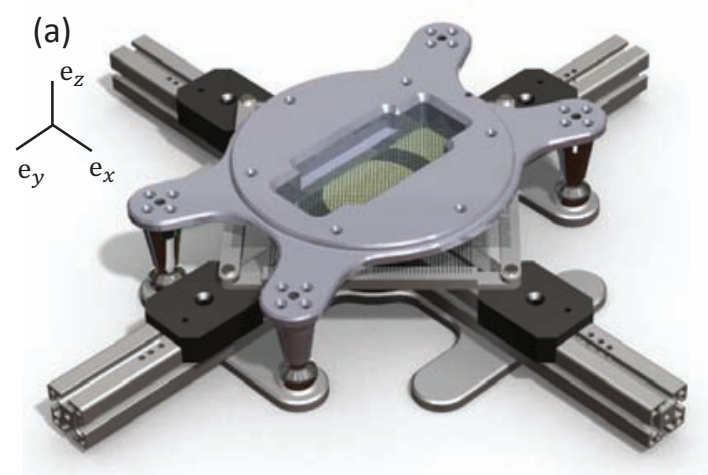

(c)

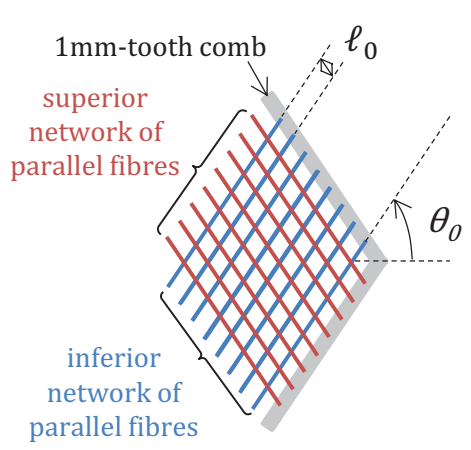

(b)

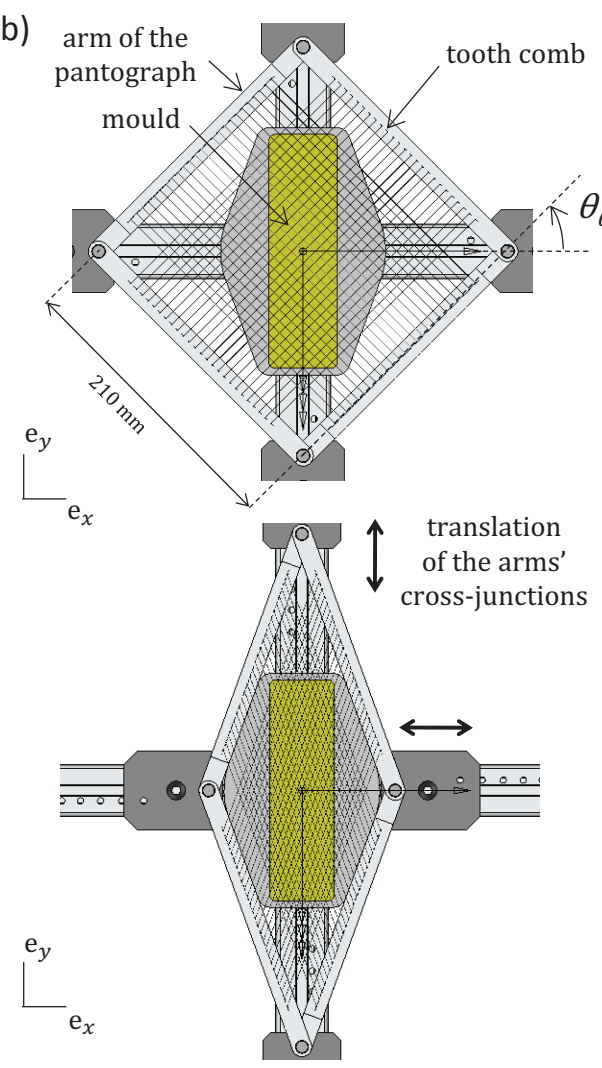

(d)
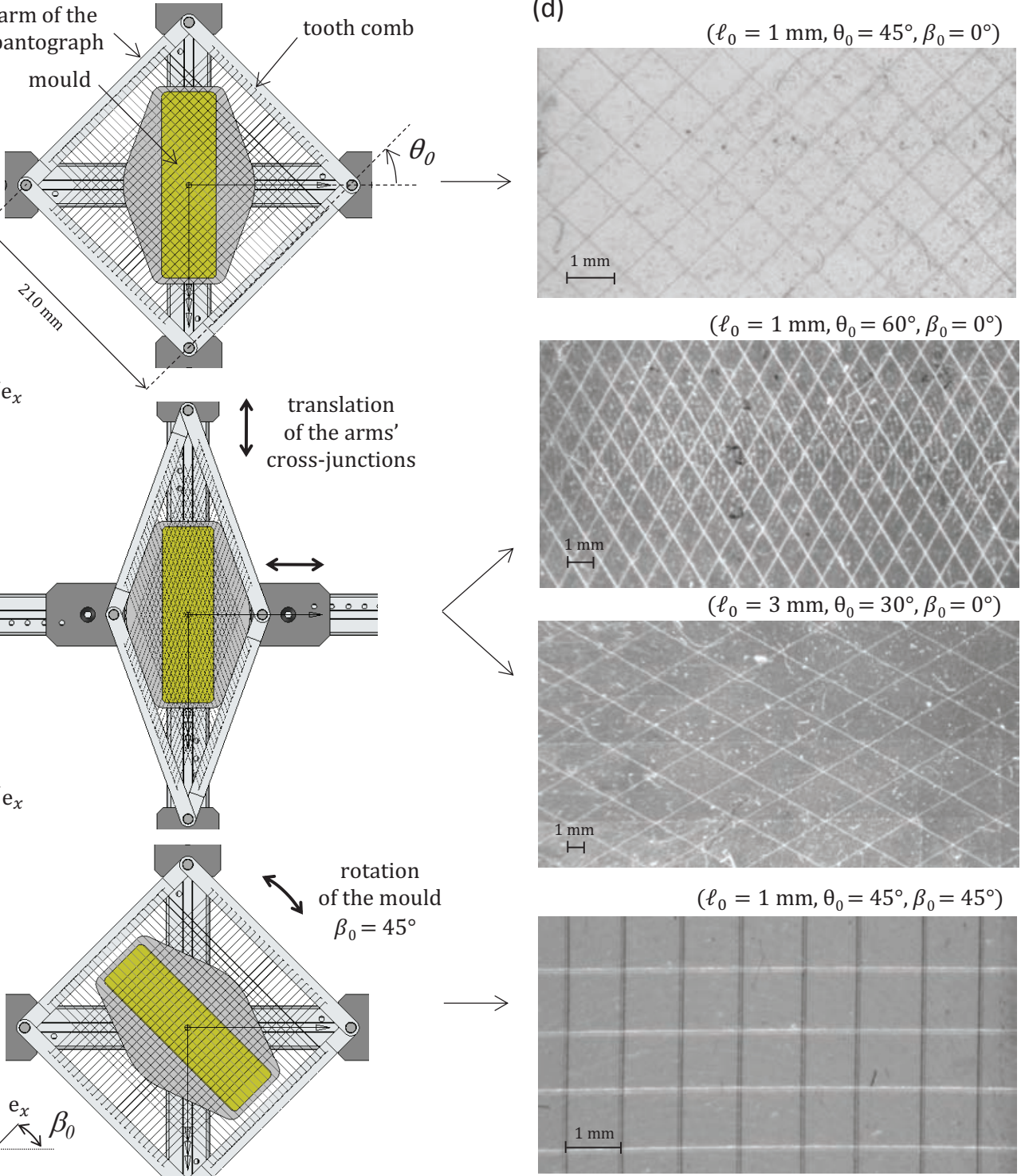

$\mathrm{e}_{y} \mathrm{e}_{x} \beta_{0}$ $\beta_{0}=45^{\circ}$

Figure 2. (a) 3D-scheme of the device designed to manufacture the composite membranes. (b) $2 \mathrm{D}$-view of the device's motion possibilities. The yellow part represents the lower part of the mould dedicated to the membrane casting (superior part of the device being not shown here). (c) Schematic zoom of a $\not \nsupseteq$-view of the pantographic structure for the lattice elaboration. (d) Pictures of some resulting composite membranes. Composites with perpendicular fibres could be created by two different manufacturing processes (see first and last rows). 
(a) $\beta_{0}=0^{\circ}$

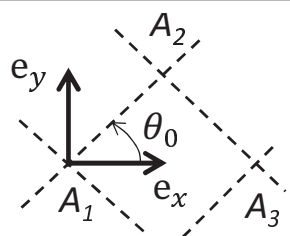

'.',

(b) $\beta_{0}=45^{\circ}$
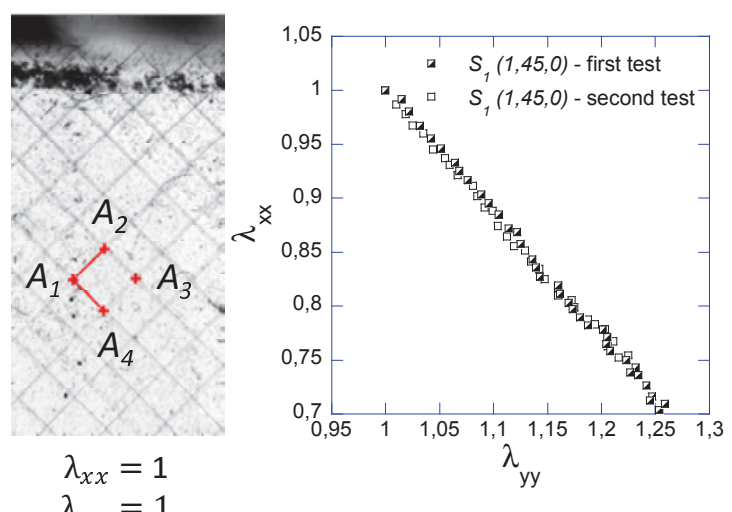

$\lambda_{x x}=1$

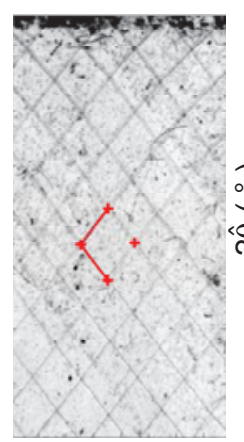

$\lambda_{x x}=0.86$

$\lambda_{y y}=1.15$

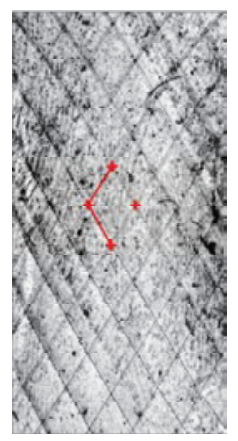

$\lambda_{x x}=0.76$

$\lambda_{y y}=1.27$
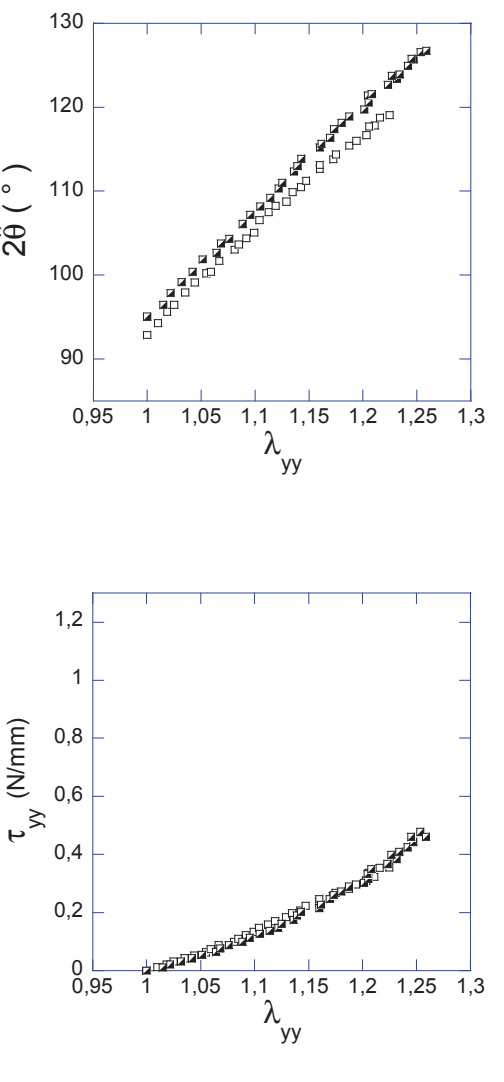
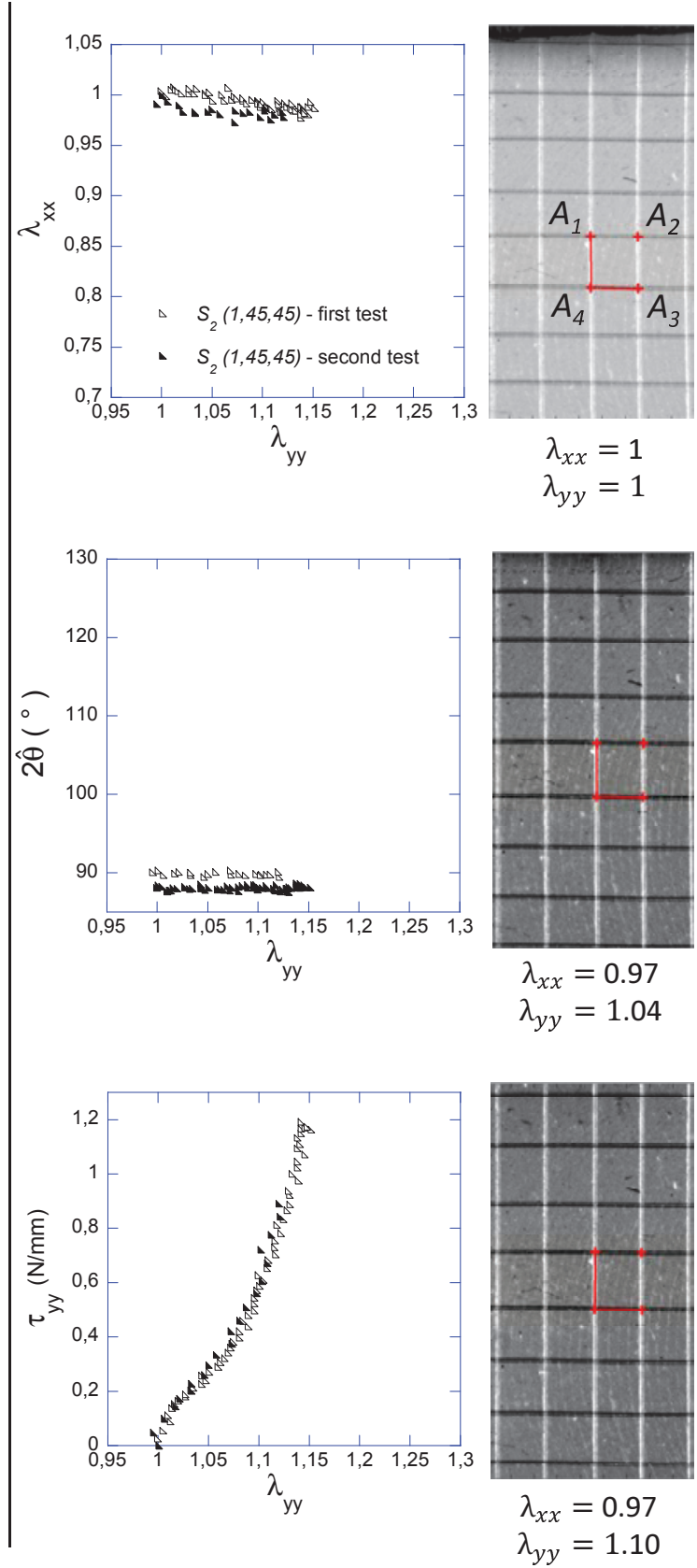

Figure 3. Typical post-processing of the measured data, using the tracking of the experimental REC nodes $A_{i}$. Illustration for two distinct loading directions $\beta_{0}$, with $S_{1}(1,45$, 0) (a) and $S_{2}(1,45,45)$ (b). 
Table 1

Dimensions and microstructural parameters of composite samples $S_{i}\left(\ell_{0}, \theta_{0}, \beta_{0}\right)$. ${ }^{\dagger}$ Values measured over all RECs of the strip's surface: mean value (minimum - maximum).

\begin{tabular}{c|ccccc}
\hline Samples & $\begin{array}{c}\overline{\ell_{0}}(\mathrm{~mm})^{\dagger} \\
\pm 0.05\end{array}$ & $\begin{array}{c}\overline{\theta_{0}}\left({ }^{\circ}\right)^{\dagger} \\
\pm 0.2\end{array}$ & $\begin{array}{c}L_{0}(\mathrm{~mm}) \\
\pm 0.02\end{array}$ & $\begin{array}{c}W_{0}(\mathrm{~mm}) \\
\pm 0.02\end{array}$ & \begin{tabular}{c}
$T_{0}(\mathrm{~mm})$ \\
\hline$S_{1}(1,45,0.02$
\end{tabular} \\
\hline$S_{2}(1,45,45)$ & $0.96(0.86-1.08)$ & $45.3(43.0-47.6)$ & 11.22 & 7.75 & 1.06 \\
\hline$S_{3}(3,45,0)$ & $2.93(2.60-3.08)$ & $45.8(43.8-48.1)$ & 28.31 & 21.12 & 0.85 \\
\hline$S_{4}(3,45,45)$ & $2.85(2.76-2.96)$ & $45.1(44.2-46.5)$ & 22.28 & 15.34 & 0.81 \\
\hline$S_{5}(1,60,0)$ & $0.95(0.79-1.10)$ & $60.4(57.6-63.5)$ & 18.04 & 6.39 & 0.93 \\
\hline$S_{6}(1,30,0)$ & $0.97(0.86-1.07)$ & $30.2(28.6-31.8)$ & 20.82 & 10.31 & 1.11 \\
\hline$S_{7}(3,60,0)$ & $2.90(2.68-3.07)$ & $60.2(57.8-62.6)$ & 47.96 & 17.54 & 1.05 \\
\hline$S_{8}(3,30,0)$ & $2.92(2.61-3.16)$ & $31.1(29.1-33.3)$ & 37.91 & 25.29 & 0.77 \\
\hline
\end{tabular}


Table 2

Differences between the target value of the initial half-angle between fibres $\theta_{0}$, the mean value measured on the whole sample surface $\overline{\theta_{0}}$, and the value measured over 4 RECs to determine the local kinematical field $\widehat{\theta_{0}}$ ( ${ }^{\dagger}$ bold values are the ones used for theoretical predictions illustrated in Figures 5 and 6 ).

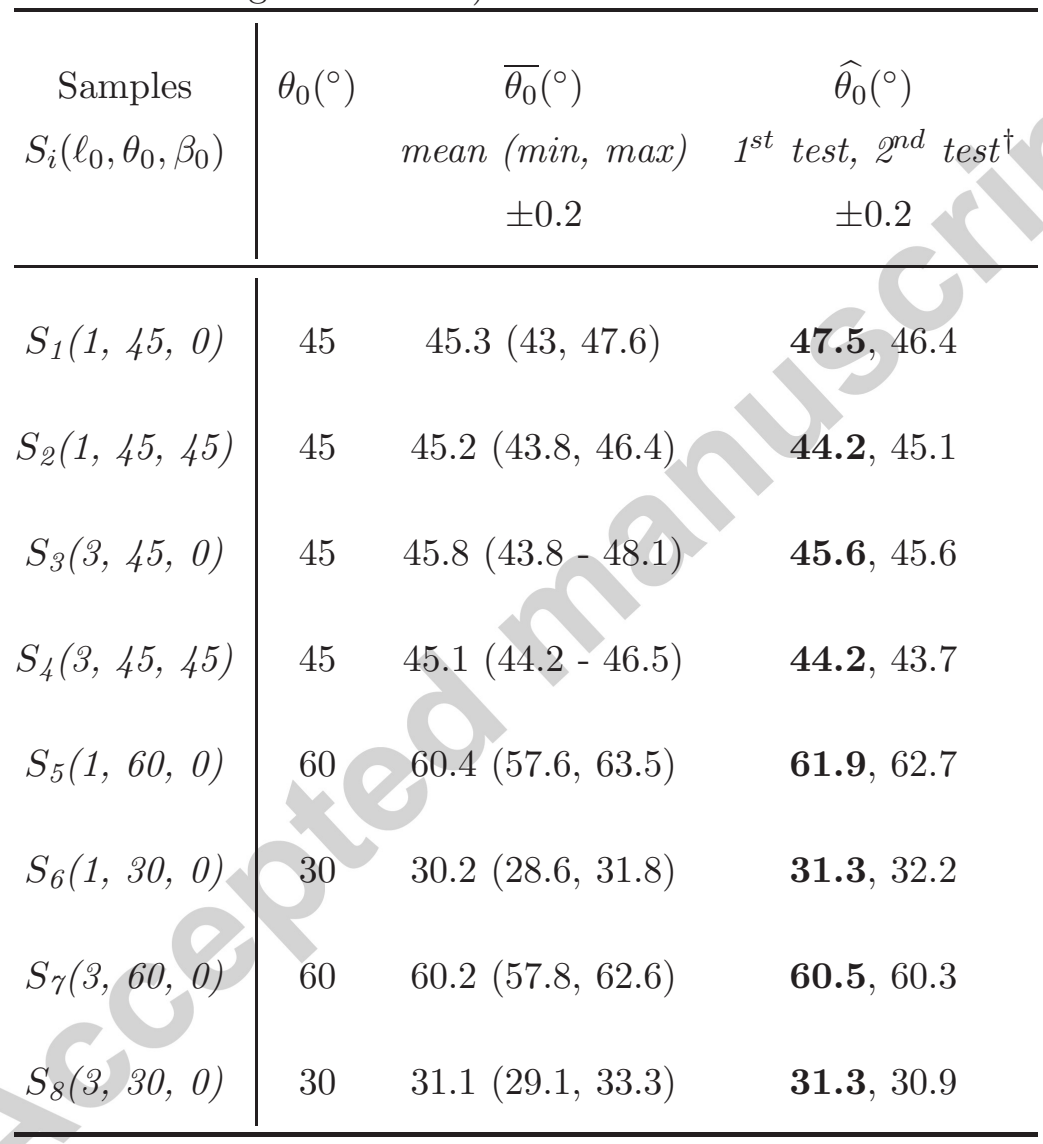



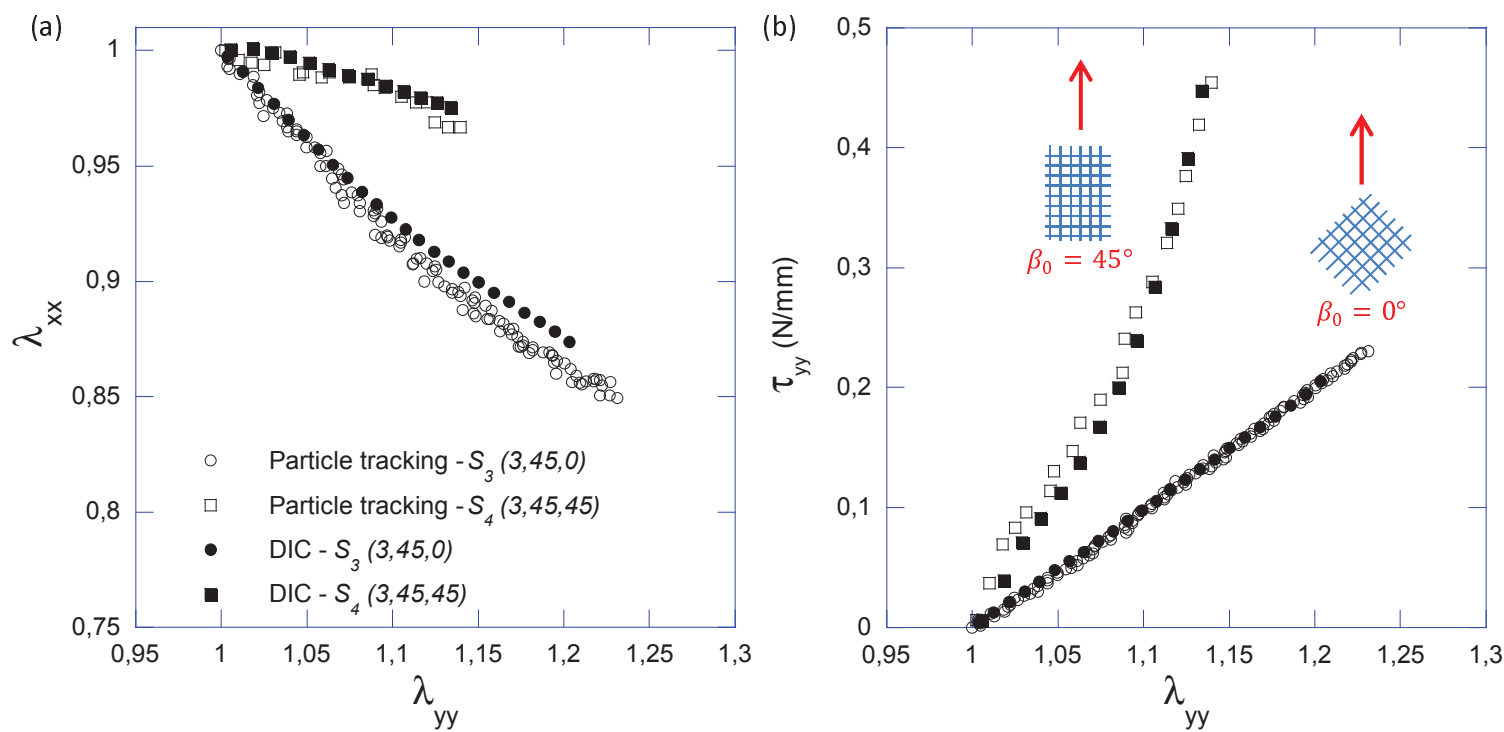

Figure 4. Comparison between global kinematics at the macroscale determined by 2D DIC and local kinematics at the microscale determined by tracking of experimental REC extremities. Illustration for two distinct loading directions, with samples $S_{3}(3,45,0)$ and $S_{4}(3,45,45)$. Same legend for (a) and (b). 

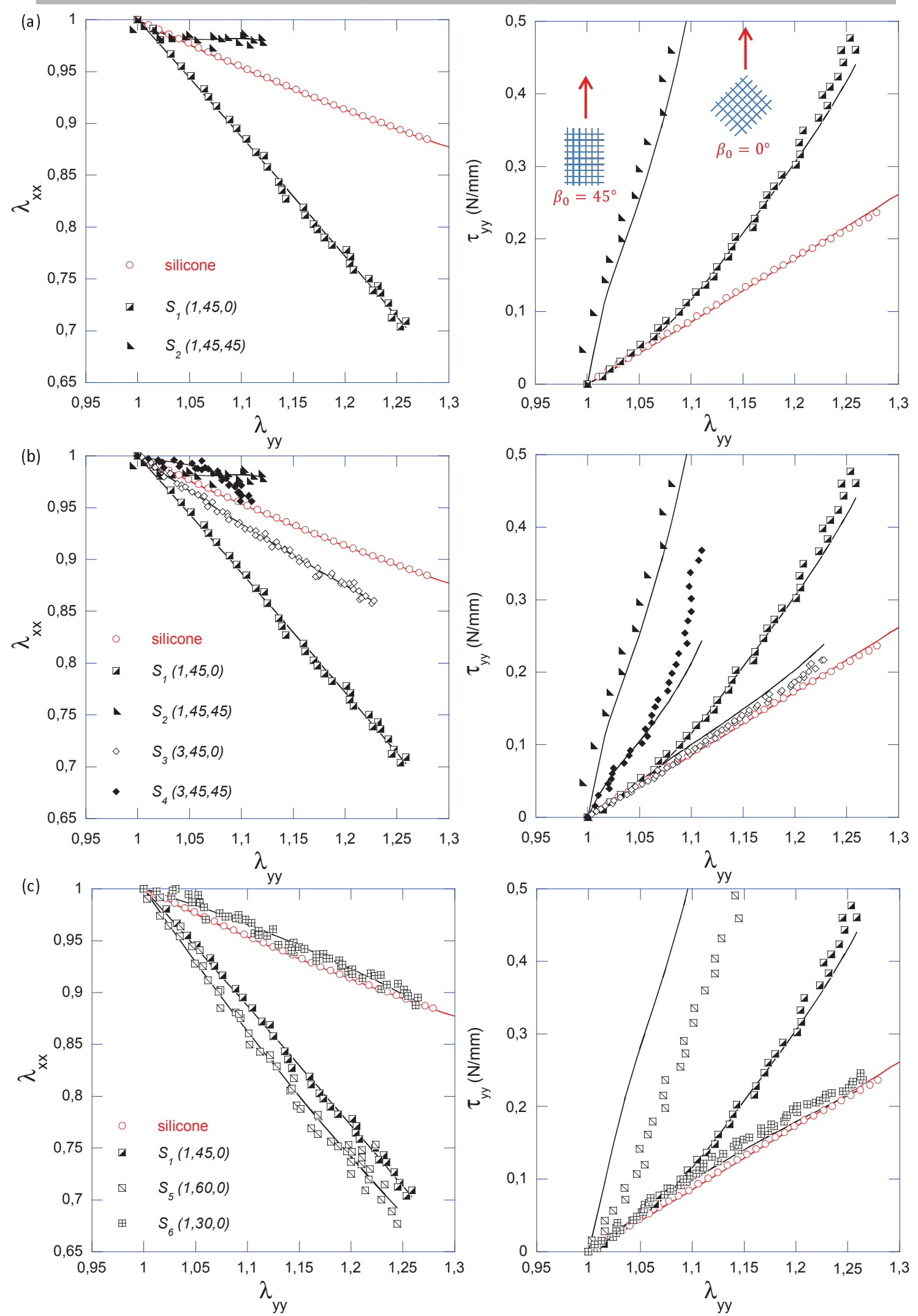

Figure 5. Influence of (a) the loading direction $\beta_{0}$, (b) the initial fibre length $\ell_{0}$ and (c) the initial half-angle between fibres $\theta_{0}$ on the mechanics of the composite membranes. Comparison between the predictions of the micro-méchanical model (solid lines) and experimental data (symbols). 

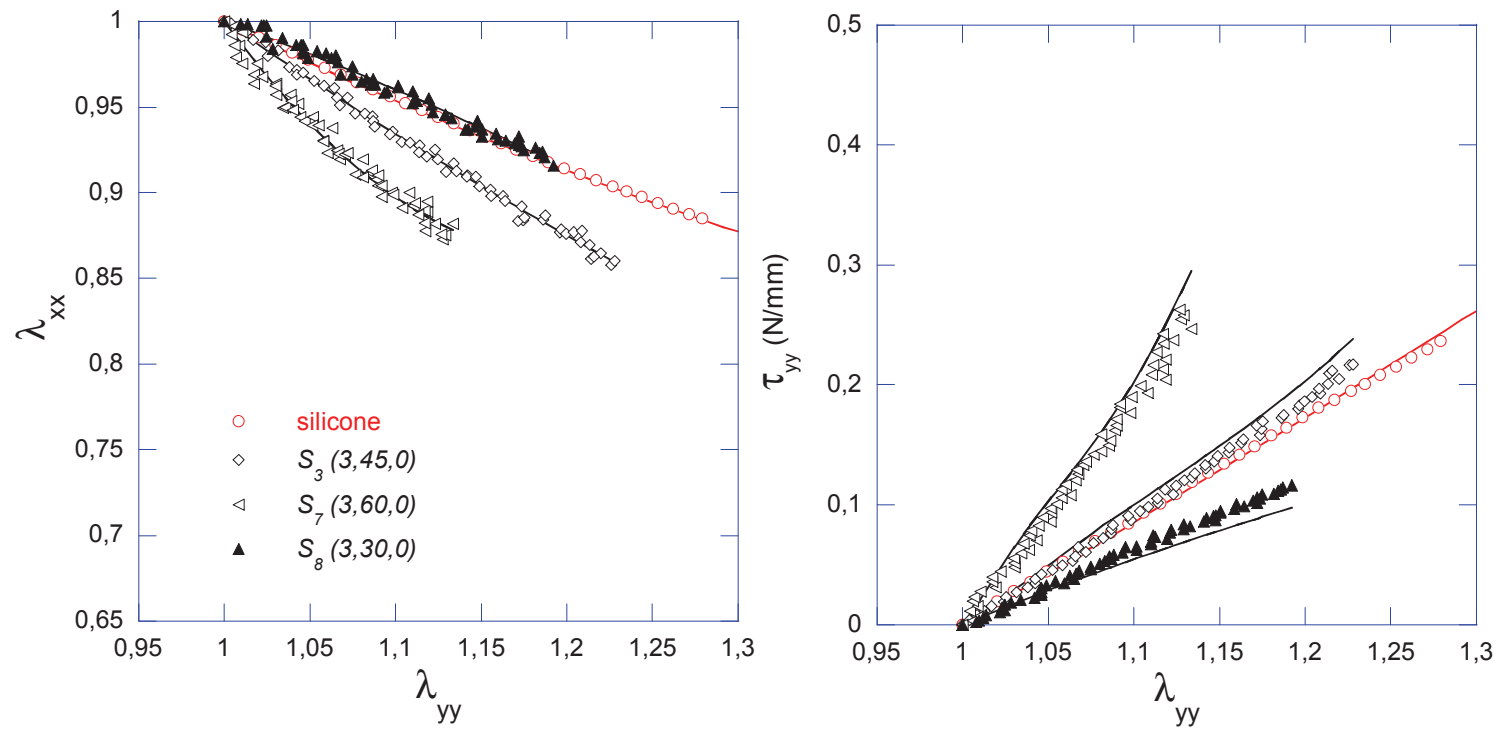

Figure 6. Same as in Figure 5 for composites with $\ell_{0}=3 \mathrm{~mm}$ and $\beta_{0}=0^{\circ}$. Influence of the initial half-angle between fibres, $\theta_{0}$. 

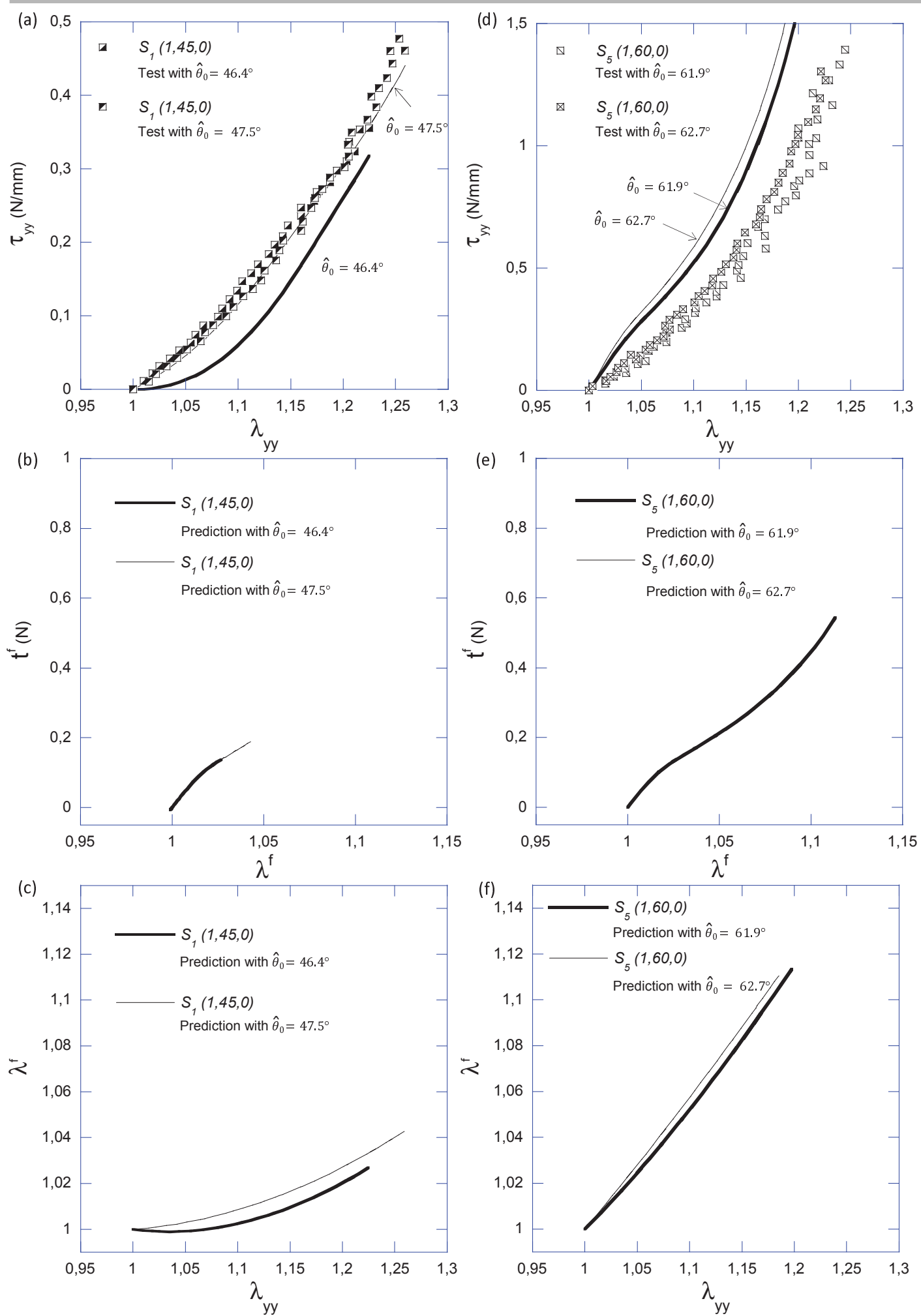

Figure 7. (a, d) Critical impact of the angle $\widehat{\theta_{0}}$ on the theoretical predictions of the composite's Cauchy tension-elongation response (solid lines). Illustration on two samples, $S_{1}(1,45,0)(\mathbf{a})$, and $S_{5}(1,60,0)(\mathbf{d})$. ( b, e) Corresponding predictions of the load within REC fibre $i(i=1,2)$, as function of fibre elongation. $(\mathbf{c}, \mathbf{f})$ Predictions of the fibre elongation, as function of the macroscopic loading in the loading direction. 